Draft Version December 24, 2019

Typeset using $\mathrm{LAT}_{\mathrm{E} X}$ preprint style in AASTeX63

\title{
Dynamical evidence for an early giant planet instability*
}

\author{
Rafael Ribeiro de Sousa, ${ }^{1,2}$ Alessandro Morbidelli, ${ }^{2}$ Sean N. Raymond, ${ }^{3}$ Andre Izidoro, ${ }^{1}$ \\ Rodney Gomes, ${ }^{4}$ ANd ERnesto Vieira Neto ${ }^{1}$ \\ ${ }^{1}$ São Paulo State University, UNESP, Campus of Guaratinguetá, Av. Dr. Ariberto Pereira da Cunha, 333 - \\ Pedregulho, Guaratinguetá - SP, 12516-410, Brazil \\ ${ }^{2}$ Laboratoire Lagrange, UMR7993, Université Côte d'Azur, CNRS, Observatoire de la Côte d'Azur, Boulevard de \\ l'Observatoire, 06304 Nice Cedex 4, France \\ ${ }^{3}$ Laboratoire dAstrophysique de Bordeaux, Univ. Bordeaux, CNRS, B18N, alle Geoffroy Saint-Hilaire, 33615 Pessac, \\ France \\ ${ }^{4}$ Observatório Nacional, Rua General José Cristino 77, CEP 20921-400, Rio de Janeiro, RJ, Brazil
}

(Received February 22, 2019; Revised December 15, 2019; Accepted December 15, 2019)

\author{
Submitted to ICARUS
}

\begin{abstract}
The dynamical structure of the Solar System can be explained by a period of orbital instability experienced by the giant planets. While a late instability was originally proposed to explain the Late Heavy Bombardment, recent work favors an early instability. Here we model the early dynamical evolution of the outer Solar System to self-consistently constrain the most likely timing of the instability. We first simulate the dynamical sculpting of the primordial outer planetesimal disk during the accretion of Uranus and Neptune from migrating planetary embryos during the gas disk phase, and determine the separation between Neptune and the inner edge of the planetesimal disk. We performed simulations with a range of (inward and outward) migration histories for Jupiter. We find that, unless Jupiter migrated inwards by 10 AU or more, the instability almost certainly happened within 100 Myr of the start of Solar System formation. There are two distinct possible instability triggers. The first is an instability that is triggered by the planets themselves, with no appreciable influence from the planetesimal disk. About half of the planetary systems that we consider have a self-triggered instability. Of those, the median instability time is $\sim 4 \mathrm{Myr}$. Among self-stable systems - where the planets are locked in a resonant chain that remains stable in the absence of a planetesimal's disk- our self-consistently sculpted planetesimal disks nonetheless trigger a giant planet instability with a median instability time of 37-62 Myr for a reasonable range of migration histories of Jupiter. The simulations that give the latest instability times are those that invoked long-range inward migration of Jupiter from 15 AU or beyond; however these simulations over-excited the inclinations of Kuiper
\end{abstract}

Corresponding author: Rafael Ribeiro de Sousa

rafanw72@gmail.com, rribeiro@oac.eu

* Article accepted for publication on December, 15th, 2019, https://authors.elsevier.com/tracking/article/details.do? aid $=113605 \& j i d=$ YICAR\&surname $=$ Ribeiro 
belt objects and are inconsistent with the present-day Solar System. We conclude on dynamical grounds that the giant planet instability is likely to have occurred early in Solar System history.

Keywords: Giant Planet instability; Planetesimals; Planet-disk interactions; Planets, migration; Solar System dynamical evolution.

\section{INTRODUCTION}

The orbital evolution of the giant planets played a central role in shaping the dynamical structure of the present-day Solar System. The so-called Nice model invokes a dynamical instability among the giant planets that was triggered by interactions with the primordial outer planetesimal disk (Tsiganis et al. 2005; Levison et al. 2011; Nesvorný \& Morbidelli 2012). There are a number of lines of circumstantial evidence that support this model. A giant planet instability can explain the capture of the Trojans of Jupiter and Neptune (Morbidelli et al. 2005; Nesvorný et al. 2013; Gomes \& Nesvorný 2016), the irregular satellites of the giant planets (Nesvorný et al. 2007, 2014) and the Kuiper belt's orbital structure (Nesvorný 2015a,b; Nesvorný \& Vokrouhlický 2016; Gomes et al. 2018) see also Nesvorný (2018) for a review.

The giant planet instability caused a cometary bombardment in the inner Solar System (Gomes et al. 2005). According to the Nice model scenario, after the gas dissipation Jupiter, Saturn, Uranus, and Neptune's gravitational interactions with a massive outer planetesimal disk eventually drove them into an unstable configuration. During the giant planet instability, the outer planetesimal disk was mostly destabilized. The asteroid belt was also strongly perturbed, removing $\sim 90 \%$ of the original asteroids (Nesvorný et al. 2017; Deienno et al. 2016, 2018; Clement et al. 2019a). As a consequence, a large number of planetesimals (both asteroids and comets) was delivered into the inner Solar System, causing a massive bombardment on the terrestrial planets and the Moon assuming that the Moon and the planets existed at this time (Gomes et al. 2005; Bottke et al. 2012). If this happened late, the instability would have had a high probability of disrupting the orbits of the terrestrial planets (Kaib \& Chambers 2016) or at least of over-exciting their orbits (Roig et al. 2016). However, an instability during terrestrial planet formation would have excited the terrestrial planets' building blocks - for instance by removing mass from Mars' feeding zone but not Earth's, simulations of terrestrial planet formation with an early giant planet instability provide a good match to the Solar System (Clement et al. 2018b, 2019a,b).

The Lunar crater record suggests an epoch of intense bombardment around 3.9 Gy ago, when impacts created the youngest basins of the Moon. These impacts took place late in the Solar System formation time line, roughly 500 - 700 My after the planets formed; this period is known as the Late Heavy Bombardment (LHB). Two hypotheses have been suggested to explain the origin of the LHB. The first claims that the LHB was due to a surge on the bombardment rate (Tera et al. 1974; Ryder 1990, 2002). This may be explained by the destabilization of the asteroid belt and trans-Neptunian disk due to a sufficiently late giant planet instability, as described above (Gomes et al. 2005). In this line of thinking the instability would have happened $\sim 500-700 \mathrm{My}$ after the planets formed. The second hypothesis is that the LHB was the tail-end of the terrestrial planet accretion, presumably due to planetesimals left-over from the main phase of planet formation (Hartmann 1975; Neukum et al. 2001; Hartmann 2003). Morbidelli et al. (2012a) showed that in this hypothesis the Moon would 
have accreted a mass that is an order of magnitude larger than that deduced from the abundance of highly siderophile elements (HSE) in its mantle (Walker 2009; Day et al. 2007; Day \& Walker 2015). But Morbidelli et al. (2018) showed that the lunar HSEs could have been sequestered into the lunar core during the crystallization of the lunar magma ocean (LMO). The HSE budget of the lunar mantle would then trace only the amount of material accreted after LMO crystallization. If the latter occurred late, as argued in Elkins-Tanton et al. (2011), the small amount of lunar mantle HSEs can be explained also in the accretion-tail hypothesis for the LHB. Therefore, both late or early instabilities may be consistent with the lunar crater record and geochemical properties.

To date it has been assumed that the giant planet instability was triggered by gravitational interactions between the giant planets and an outer planetesimal disk (Tsiganis et al. 2005; Morbidelli et al. 2007; Levison et al. 2011; Deienno et al. 2017), (see Thommes et al. (2002) for an exception). However, this was not necessarily the case. Below we demonstrate that the instability may instead have been self-triggered by the giant planets themselves. Upon dissipation of their natal planet-forming disks, giant planets emerge on marginally stable (and often quickly unstable) configurations (e.g., Moeckel et al. 2008; Matsumura et al. 2010). A large number of studies have simulated self-triggered instabilities, mainly in the context of explaining the eccentricity distribution of giant exoplanets (e.g., review by Davies et al. 2014). Instabilities arising as gravitational jostling between planets cause their orbits to intersect, leading to a phase of close encounters (often called 'planet-planet scattering'), which typically leads to the ejection of one or more planets into interstellar space (Rasio \& Ford 1996; Weidenschilling \& Marzari 1996; Lin \& Ida 1997; Adams \& Laughlin 2003). Simulations of self-triggered instabilities find that 1) more closely-packed sets of planets generally become unstable on shorter timescales (Chambers et al. 1996; Marzari \& Weidenschilling 2002; Chatterjee et al. 2008) and 2) marginally-stable systems with outer planetesimal disks that do become unstable tend to do so relatively quickly, with a median instability time of $10^{5}$ years but a tail extending out to hundreds of Myr (Raymond et al. 2010, 2011).

Interactions with the planetesimal disk remains a possible instability trigger if the giant planets emerged from the gaseous disk on long-term stable orbits. The timing of such an instability is set by the distance between the outermost planet (presumably Neptune) and the inner edge of the primordial trans-Neptunian planetesimal disk (Gomes et al. 2005; Levison et al. 2011). Gomes et al. (2005) argued that the planetesimal disk should only contain particles with dynamical lifetimes (times required to encounter a planet) longer than the lifetime of the gaseous disk (a few My e.g., Haisch et al. 2001; Hillenbrand 2008; Pascucci et al. 2009). Planetesimals with short dynamical lifetimes should have been removed with little effect on the dynamical evolution of the planets because planet-gas interactions dominate over the planet-planetesimal interactions (Capobianco et al. 2011).

Gomes et al. (2005) thus argued for a roughly 1 AU-wide gap between Neptune's early orbit and the inner edge of the planetesimal disk and found that the giant planet instability occurred in this case between 200 Myr and 1 Gyr, roughly at the time expected for the LHB. Levison et al. (2011) considered 4 resonant giant planets where the inner edge of the planetesimal disk was several AUs beyond the orbit of Neptune (the outermost planet). They showed that viscous stirring (due to the disk's self-gravity, assuming the presence of a thousand Pluto-mass objects) leads to an exchange of energy between the planet and disk particles even in the absence of close encounters. This energy exchange proceeds at a very slow pace and the system crosses many weak secular resonances that lead to instability on a timescale consistent with the Late Heavy Bombardment. Deienno et al. (2017) 
simulated the outer Solar System's evolution assuming the presence of an additional primordial ice giant (Nesvorný 2011) and testing a variety of orbital configurations of the 5 giant planets. They found that a late instability is possible for a specific minimum distance between the inner edge of the disk and Neptune. However, in some cases, the timescale of instability was dependent on the numerical resolution of the planetesimal disk (mass and number of the planetesimals). Of course, the real primordial planetesimal disk was sculpted by the growth and migration of the giant planets. If the giant planets or their precursors migrated inward from farther out in the gaseous disk (e.g, Bitsch et al. (2015)), this could plausibly have created a significant gap between the outermost ice giant and the primordial planetesimal disk. On the other hand, planetesimals excited onto eccentric orbits by the forming/migrating planets may have their orbits re-circularized at perihelion distance by gas drag (e.g. Raymond \& Izidoro 2017), narrowing the gap between the planets and planetesimal disk.

The goal of this paper is to constrain the timing of the giant planet instability by simulating interactions between the giant planets and a sculpted outer planetesimal disk. To do this, we need a reliable model of the growth and early evolution of the giant planets' orbits, in particular for the ice giants. Indeed, understanding the accretion of Uranus and Neptune is a long-standing problem in Solar System formation. Early studies showed that their growth by planetesimal accretion takes longer than the gas disk lifetime (Safronov 1969; Levison \& Stewart 2001; Thommes \& Lissauer 2003), even at $10-15 \mathrm{AU}$ (Levison et al. 2010). The accretion of mm- to cm-sized "pebbles" aerodynamically drifting through the disk has been shown to drastically accelerate core growth (Lambrechts \& Johansen 2012, 2014). For reasonable pebble fluxes cores of 10 - 20 Earth masses can form within the gas disk lifetime (Lambrechts et al. 2014) even when gravitational interactions among the growing protoplanets are accounted for Levison et al. (2015). Yet pebble accretion should produce planets with zero obliquity (Dones \& Tremaine 1993; Johansen \& Lacerda 2010). The 3 degree obliquity of Jupiter and the 26-degrees obliquity of Saturn can be explained by spin-orbit resonances with Uranus and Neptune, respectively (Ward \& Hamilton 2004; Hamilton \& Ward 2004; Boué et al. 2009; Vokrouhlický \& Nesvorný 2015; Brasser \& Lee 2015). However, Uranus and Neptune's obliquities of 98 and 30 degrees, respectively, are thought to be the result of giant collisions during their formation (Slattery et al. 1992; Boué \& Laskar 2010; Morbidelli et al. 2012b; Jakubík et al. 2012; Kegerreis et al. 2018).

Izidoro et al. (2015a) proposed that the ice giants formed in two steps. By the time Jupiter and Saturn had undergone rapid gas accretion to become giants, pebble accretion had produced a system of protoplanets of $\sim 5$ Earth masses, comparable in mass to most super-Earths (e.g., Mayor et al. 2011; Batalha et al. 2013; Marcy et al. 2014; Wolfgang et al. 2016). These protoplanets migrated inward in the Type-I regime due to tidal torques from the gas disk (Goldreich \& Tremaine 1980; Ward 1986; Tanaka et al. 2002). While it is unclear whether Jupiter and Saturn at this point would have been migrating inward, outward or have roughly stationary orbits (Masset \& Snellgrove 2001; Morbidelli \& Crida 2007; Pierens \& Nelson 2008; Pierens \& Raymond 2011; Raymond \& Morbidelli 2014; Pierens et al. 2014a), the gas giants' migration was certainly slower than that of the protoplanets for typical disk viscosities. In addition, the co-presence of Jupiter and Saturn has the effect to slowdown, stop or reverse, the resonant migration of these two planets, depending on the aspect ratio of the disk (Crida \& Morbidelli 2007). 
The protoplanets cannot generally migrate past Jupiter and Saturn's orbits; rather, the gas giants act as a migration barrier (Izidoro et al. 2015b). Protoplanets become trapped in mean motion resonances with Saturn, and successive protoplanets form chains of mean motion resonances. As more protoplanets migrate inward, the resonant chain is destabilized, leading to giant (obliquitygenerating) collisions and another phase of migration into resonant chains. Izidoro et al. (2015a) showed that this process typically produces $2-3$ ice giants with masses comparable to those of Uranus and Neptune in resonant chains that include Jupiter and Saturn.

We constrain the timing of the giant planet instability in two ways. First, we simulate the dynamical evolution of the successful ice giant formation systems produced by Izidoro et al. (2015a). Second, we generate planetesimal disks that are dynamically consistent with the growth and migration of the giant planets, again using the framework of Izidoro et al. (2015a). Our paper is laid out as follows. In $\S 2.2$ we discuss how we simulated the evolution of the primordial planetesimal disk during the growth of Uranus and Neptune during the gas disk phase. We also tested different migration histories for Jupiter. In $\S 3$ we simulate the giant planet instability under two different assumptions. We first show that the giant planets in successful runs from Izidoro et al. (2015a) are often self-unstable after the dissipation of the gaseous disk. Next, we show that even planetary systems that would be long-term stable without any influence of the planetesimal disk do become quickly unstable under the influence of planetesimal disks consistent with the growth and dynamical evolution of the giant planets. This result holds for all migration histories of Jupiter in which the planet migrated inwards by less than 10 AU. In $\S 4$ we discuss caveats to our study. We present our conclusions in $\S 5$.

\section{SCULPTING OF THE PLANETESIMAL DISK DURING THE ICE GIANTS' GROWTH}

\subsection{Model of Izidoro et al. (2015)}

In this section, we focus on the best simulation of Izidoro et al. (2015a) in which three Neptunemass planets formed and no initial protoplanets were left behind in the Solar System. This final outcome is consistent with the most modern version of the Nice model (Nesvorný \& Morbidelli 2012), which drastically increases the success rate in reproducing the outer Solar System by invoking the existence of an additional primordial Neptune-mass planet. The Izidoro et al. (2015a) simulation is illustrated in Figure 1. In panel (a), we show the initial semi major axes and eccentricities of the planetary system which contains Jupiter (at 3.5 AU), Saturn (at $4.58 \mathrm{AU}$ ) and a collection of 11 protoplanets distributed with semi major axis in a range from 6 to 26 AU with masses between 3 to 9 Earth's mass. All bodies started in circular orbits. In panel (b), we show the final semi major axis and eccentricity of Jupiter, Saturn, Uranus, Neptune and a fifth comparable-mass planet at the end of the 3 Mys lifetime of the protoplanetary disk of gas. This simulation reproduces the real masses of Uranus and Neptune, around of 15 Earth's masses. The dynamical evolution of this planetary system is showed in panel (c). The planetary embryos migrated inward because of the presence of the gas and were trapped in mean-motion resonances with the giant planets. The mutual gravitational interaction among the planetary embryos eventually broke the resonant chains and the system became dynamically unstable. During this phase, the planetary embryos were scattered by mutual close encounters and encounters with the giant planets. Some objects were ejected from the system while other objects merged, building the three final massive planets.

For the gas disk, Izidoro et al. (2015a) used the $1 \mathrm{D}$ radial density distribution obtained from hydrodynamical simulations that made use of the prescription of Shakura \& Sunyaev (1973) using 


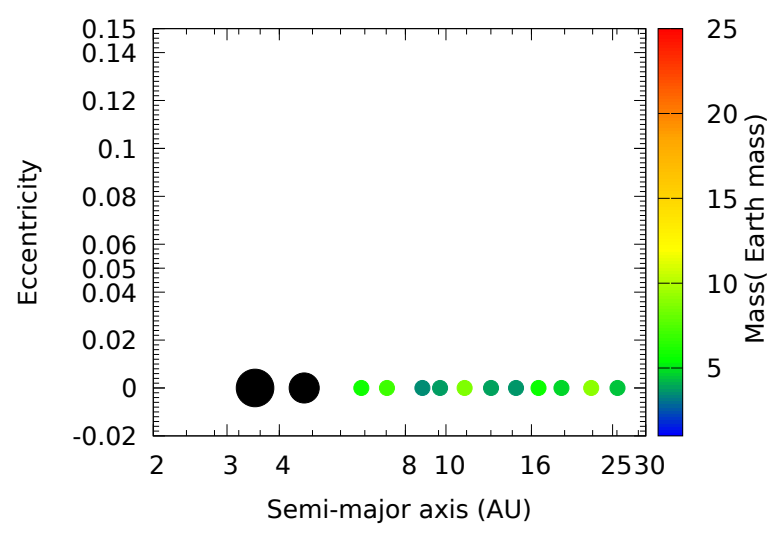

(a)

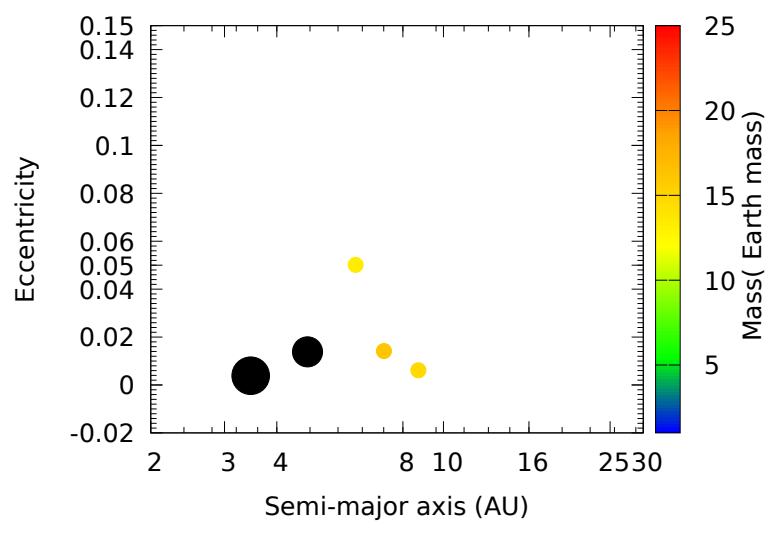

(b)

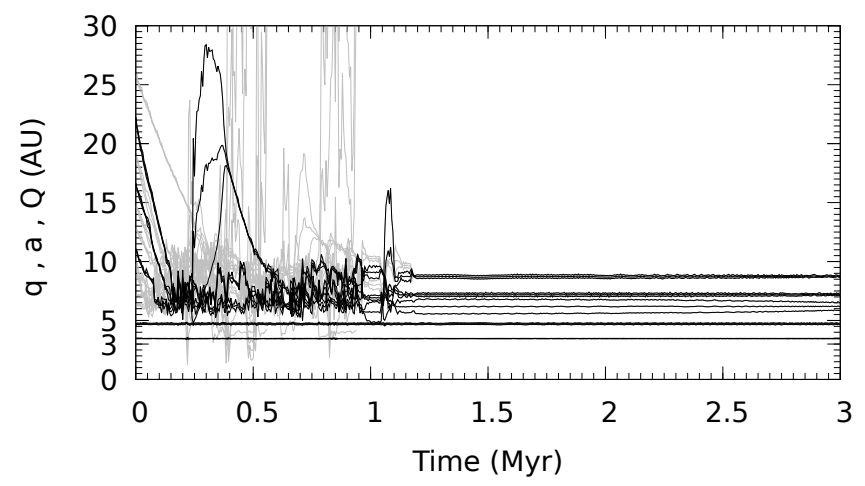

(c)

Figure 1. Panels (a) and (b) are two snapshots which represent the eccentricity as a function of semimajor axis of the system with Jupiter, Saturn and a collection of planetary embryos (initial (a) and final (b)) of the considered Izidoro et al. (2015a) simulation. Note that 3 planets are produced beyond Saturn, but one will be ejected during the planet instability (Nesvorný \& Morbidelli 2012). Panel (c) represents a complete evolution in pericenter (q), semimajor axis (a), and apocenter (Q) of the same simulation. Black lines correspond to the planets and gray lines correspond to the planetary embryos.

the value of alpha of 0.002 (more details about the disk are given in the section 2.2.1). To include the effects of type I migration for the planetary embryos, they followed Paardekooper \& Papaloizou (2008) invoking the locally isothermal approximation to describe the thermodynamics of the disk. In this case, the Lindblad and the coorbital torques contributions can be written in terms of the negative of the local gas surface density and temperature gradients. To mimic the effects of eccentricity and inclination damping in their simulations, they followed Papaloizou \& Larwood (2000) and Cresswell \& Nelson (2006, 2008). Note that in Fig. 1 the ice giants show moderate orbital eccentricity. This is due to the way that the planets are assembled together into mean motion ressonances and about the disks properties (eccentricity damping, aspect ratio of the disk, to cite a few).

\subsection{Methods}

We used N-body simulations conducted in the framework of the study on the formation of Uranus and Neptune of Izidoro et al. (2015a). We interpolated the orbital elements of all massive bodies 
(Jupiter, Saturn and the proto-planets) from the best simulation of Izidoro et al. (2015a) using spline functions. We then used this forced interpolated evolution of the massive bodies (perturbers) in our simulations of planetesimal evolution. In addition to the perturbers, we included also the effects of a gaseous protoplanetary disk on the planetesimals (gas drag and dynamical friction) as detailed in section 2.2.1. For consistency, we adopted the surface density for the gas used by Izidoro et al. (2015a) to compute the migration and tidal damping rates on the proto-planets.

The simulations of Izidoro et al. (2015a) assumed Jupiter and Saturn on fixed orbits at 3.5 AU and 4.58 AU, respectively, motivated by the initial conditions of the Grand Tack model (Walsh et al. 2011). However, the actual migration history of Jupiter and Saturn is not well known, Bitsch et al. (2015), for example, using a disc evolution and pebble accretion, claimed that a giant planet ending at $5 \mathrm{AU}$ can only form from a seed initially located beyond $20 \mathrm{AU}$. We expect that, by controlling the migration of the proto-planets precursor of Uranus and Neptune, the migration of the Jupiter and Saturn plays an important role in the evolution of the planetesimals disk and its final structure. Thus, we scale the evolution of Saturn and the proto-planets in Izidoro et al. (2015a) simulation relatively to the semi-major axis of Jupiter and we adopt different migration histories for Jupiter in different simulations of the planetesimal disk evolution (See in Sect. 2.2.2 for a complete explanation). Obviously, the time is also rescaled with the orbital radius, so that the orbital periods of all massive bodies obey the Kepler law.

We consider three cases of inward migration of Jupiter from 20 AU, 15 AU, and 10 AU to 5 AU, one case of outward migration from $2 \mathrm{AU}$ to $5 \mathrm{AU}$ and one case where the orbit of Jupiter is fixed at $5 \mathrm{AU}$. The interpolation of Saturn and the proto-planets evolution in rescaled coordinates and its implementation with an imposed Jupiter's migration are detailed in Sect. 2.2.2, as well as the rescaling of the gas properties. This procedure has the merit that each simulation implements the exact same evolution of the embryos (which would not have been the case if we had simulated the initial conditions of Izidoro et al. (2015a) for each Jupiter's migration history), which highlights how the final structure of the planetesimal disk depends on the giant planets migration range, removing stochastic effects.

We re-run 4 times each simulation with a different planetesimal size of $1 \mathrm{~km}, 10 \mathrm{~km}, 100 \mathrm{~km}$ and $1000 \mathrm{~km}$, each a bulk density of $3.0 \mathrm{~g} / \mathrm{cm}^{3}$ in a total of 4000 planetesimals. The planetesimals are initially distributed beyond the orbits of the giant planets, but throughout the region occupied by the proto-planets and up to $60 \mathrm{AU}$. Thus, the planetesimals initial semi-major axis range depends of the initial position of Jupiter. The eccentricities and inclinations of the planetesimal disk are initially chosen as $10^{-3}$. Their argument of pericenter and longitude of ascending node are randomly selected between 0 and 360 degrees.

We used the REBOUND code (Rein \& Liu 2012; Rein \& Spiegel 2015; Rein \& Tamayo 2015) to perform the N-body simulations of the planetesimals interacting with the Sun, Jupiter, Saturn and planetary embryos interpolated from the output of Izidoro et al. (2015a) simulation, and with the gas of the protoplanetary disk. In our simulations, during the gas disk phase planetesimals are assumed to be non-interacting test particles.

\subsubsection{Interactions of planetesimals with the gaseous protoplanetary disk}

As explained in Izidoro et al. (2015a), although real hydrodynamical simulations would be ideal to study the problem, they would be impracticable given the multi-Myrs timescales and the number of 

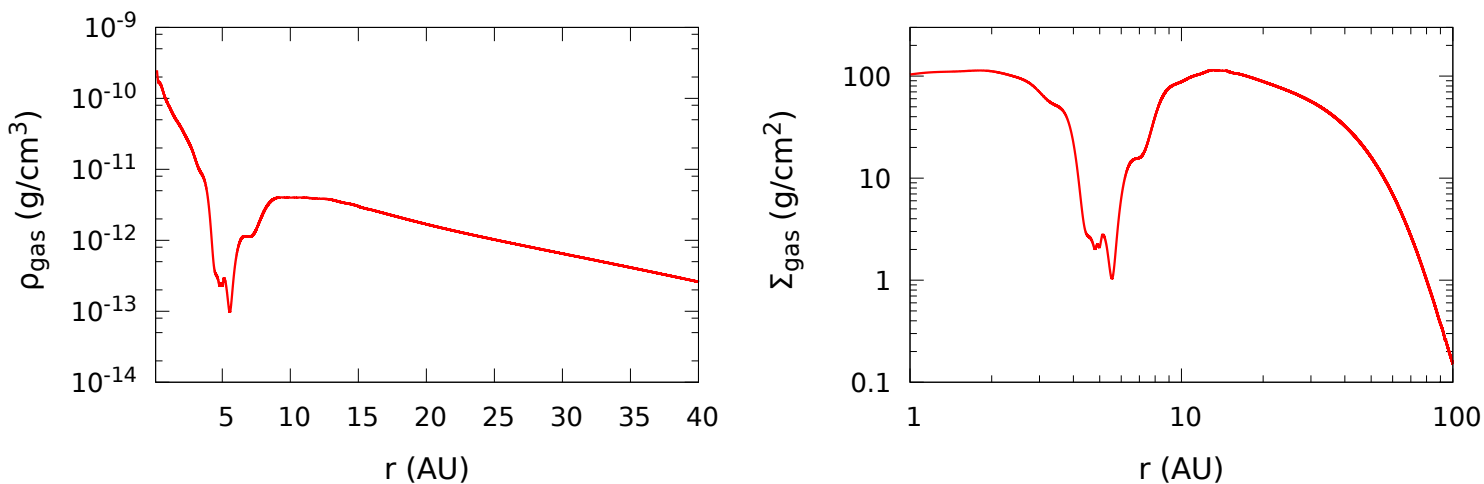

Figure 2. The protoplanetary disk profile at $t=0$ from hydrodynamical simulations in Izidoro et al. (2015a). The left panel shows the gas volumetric density and the right panel shows the surface density.

bodies involved. Thus, the gas density is used to compute synthetic forces acting on the planetesimals, and then integrating the evolution of the system with a N-body code.

From the simulation of Izidoro et al. (2015a) we have the surface density of the disk $\Sigma(r, t)$, which accounts for the gaps opened by Jupiter and Saturn in a disk as well as the partial depletion of the inner disk. This profile had been calculated from a hydrodynamical simulation, starting from a disk with initial surface density $\Sigma(r)=1,000 \mathrm{~g} / \mathrm{cm}^{2}(A U / r)$, (see right panel of Fig. 2) and assuming a uniform decay over time with $\exp \left(-t / \tau_{\text {gas }}\right)$. We also adopt from Izidoro et al. (2015a) the aspect ratio of the disk

$$
h=\frac{H(r)}{r}=0.033 r^{0.25},
$$

where $H(r)$ is the pressure scale height at radius $r$. Thus, the density of the gas has a $z$-distribution given by

$$
\rho(r, z, t)=\frac{\Sigma(r, t)}{\sqrt{2 \pi} H(r)} \exp \left(-\frac{z^{2}}{2 H^{2}}\right),
$$

which is shown in the left panel of Figure 2. In the hydrodynamical simulations, the disk viscous stress was modeled using the standard "alpha" prescription (Shakura \& Sunyaev 1973). The disk viscosity is given by:

$$
\nu=\alpha c_{s} H
$$

where, $c_{s}$ is the isothermal sound speed and the value of $\alpha$ is 0.002 in Izidoro et al. (2015)'s simulations.

The sub-keplerian velocity of the gas in the mid-plane was also read from the hydrodynamical simulations and includes the pressure gradient effect, consistent with the profiles of $\Sigma(r)$ and $H(r)$ reported above. Section 2.2.2 will discuss how all these quantities are rescaled when Jupiter is assumed to be on an orbit with a different semi-major axis.

With this information, we have all quantities needed to compute the gas drag effects on our planetesimals. The aerodynamic gas drag force on a particle moving in a gas disk environment is expressed in function of its shape, size, velocity and gas conditions. In the particular case of spherical body with radius $R$, the drag force is in a direction opposite to the particle velocity and could be expressed by:

$$
\vec{F}_{D}=-\frac{1}{2} C_{D} \pi R^{2} \rho_{g} v_{r e l} \vec{v}_{r e l}
$$


where $C_{D}$ is the drag coefficient and $\vec{v}_{r e l}$ is the vector relative velocity between the gas and the planetesimal. The drag coefficient for a spherical object is a function of the Reynolds number $\left(R_{e}\right)$ which is a measure of the turbulence of the gas in the wake of a planetesimal, the Mach Number $(M)$ and Knudsen number $(K)$. To evaluate the drag coefficient we used the approach of Brasser et al. (2007) where they estimated the values of $M, K$ and $R_{e}$ as a function of planetesimal's size and velocity.

Grishin \& Perets (2015) showed that for planetesimals within the mass range of $m \sim 10^{21}-10^{25} \mathrm{~g}$ there is another planetesimal-gas interaction, possibly dominating over gas-drag. This force is known as gas dynamical friction (GDF) and considering the same approach of Grishin \& Perets (2015), the GDF force is given by:

$$
\vec{F}_{G D F}=-\frac{4 \pi G^{2} m^{2} \rho_{g}}{v_{r e l}^{3}} \vec{v}_{r e l} I(M),
$$

$I(M)$ is a dimensionless factor depending on the Mach number (M) and it is given by:

- If $M<1$ then:

$$
I(M)=\frac{1}{2} \ln \left(\frac{1+M}{1-M}\right)-M
$$

- If $M>1$ then:

$$
I(M)=\frac{1}{2} \ln \left(1-\frac{1}{M^{2}}\right)+\ln \left(\frac{v_{r e l} t}{R}\right)
$$

We show in Figure 3 the results of the effects of the aerodynamic gas drag in the evolution of two test particles of a radius of $2 \mathrm{~km}$ using the gas profile of Grishin \& Perets (2015), for comparison with that work. The first particle (blue) has 10 degrees of inclination and eccentricity equal to 0 . The second particle has eccentricity equal to 0.2 and it has a planar orbit (red). The eccentric particle moves faster than the local gas when it is at pericenter. As a consequence, the drag decreases the apocenter distance of the particle. At apocenter, the particle moves slower than the local gas so that the drag increases the pericenter distance. The orbit will circularize at a distance between the two extremes, which depends on the radial profile of the gas since the latter governs how much damping occurs near perihelion vs. aphelion. We can see that $q$ decreases over time for the orbit initially circular and inclined, while it increases for the orbit initially eccentric and planar (see also in Brasser et al. (2007)).

We show in Figure 4 the effects of the gas dynamical friction on particles with mass of $10^{22} \mathrm{~kg}$ and with different eccentricities. We found the same results of Grishin \& Perets (2015) (aerodynamic gas drag is neglected in these tests): particles with initial low eccentricities circularized much faster than high eccentricity orbits. There are two regimes of radial drift that depend on the eccentricities: an exponential decay in semi major axis before the circularization of the orbit and a linear decay when the orbits of the particles are circular ${ }^{1}$.

${ }^{1}$ Although Grishin \& Perets (2015) have found an exponential decay when the orbits of the particles are circular, this seems to be due to a mistake in their calculation of the sound speed of the gas (Grishin and Perets, private communication). 

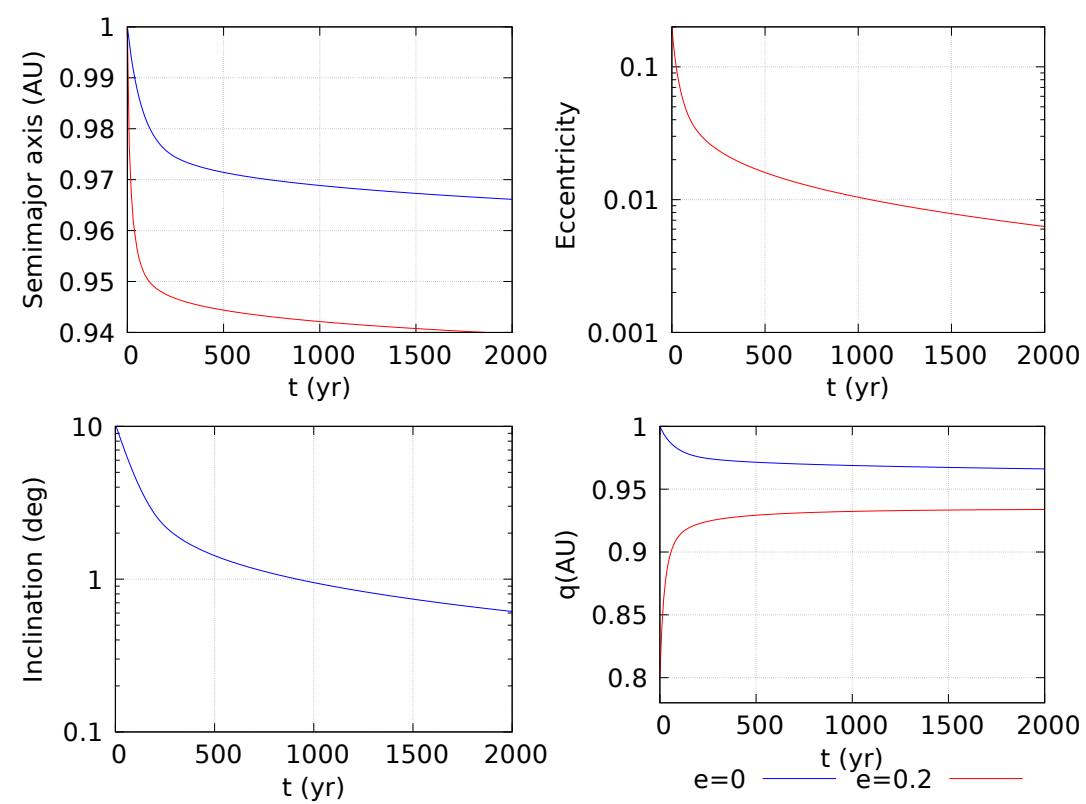

Figure 3. A summary of the action of the gas drag on a planetesimal's orbital elements. The blue solid line shows a first particle with initial eccentricity and inclination equal to 0 and 10 degrees, respectively. The red solid line shows a second particle with initial eccentricity equal to 0.2 and a planar orbit. Going clockwise from the top-left the orbital elements are the semi major axis, eccentricity, perihelion and inclination ploted in function of the time.

In Fig. 5 we show a simulation of the set of particles with different sizes under effects of the aerodynamic gas drag and dynamical friction gas drag. For this simulation, we used the surface density of the disk presented in Fig. 2. The density of the particles is $3 \mathrm{~g} / \mathrm{cm}^{3}$ and minimum particle radius $\left(R_{p}\right)$ is $1 \mathrm{~km}$ (black lines) whereas maximum particle radius is $500 \mathrm{~km}$ (purple lines). The orbits of the particles have initial eccentricity of 0.8 , initial semi-major axis of $40 \mathrm{AU}$ and initial inclination of 8.6 degrees. We observed that km-size planetesimals present a more significative radial drift, eccentricity and inclination damping than $100 \mathrm{~km}$-size planetesimals. It shows that the effects of the aerodynamic gas drag are more pronounced than dynamical friction effects. We will show in section 2.3, that the final dynamical state of the primordial planetesimal disk under the influence of the Jupiter, Saturn, planetary embryos and the gas drags is planetesimal size dependent. With a dominant aerodynamic drag, there is strong eccentricity and inclination damping in favor of km-sized planetesimals, which makes them a dynamically cold configuration.

Note that we do not need to implement the tidal forces of the disk of gas on the proto-planets, because we take their evolution from the output of the Izidoro et al. (2015a) simulation, which already implemented these forces.

\subsubsection{Interpolation of Uranus and Neptune accretion}

In this section, we present how we interpoled one successful simulation of Izidoro et al. (2015a), which we take as reference. For the interpolation we used cubic splines. We interpolate all orbital elements between two successive outputs. The time-resolution of the output in Izidoro et al. (2015a) simulation is 5,000 y. Because this exceeds the orbital period of the bodies, we calculate the number 

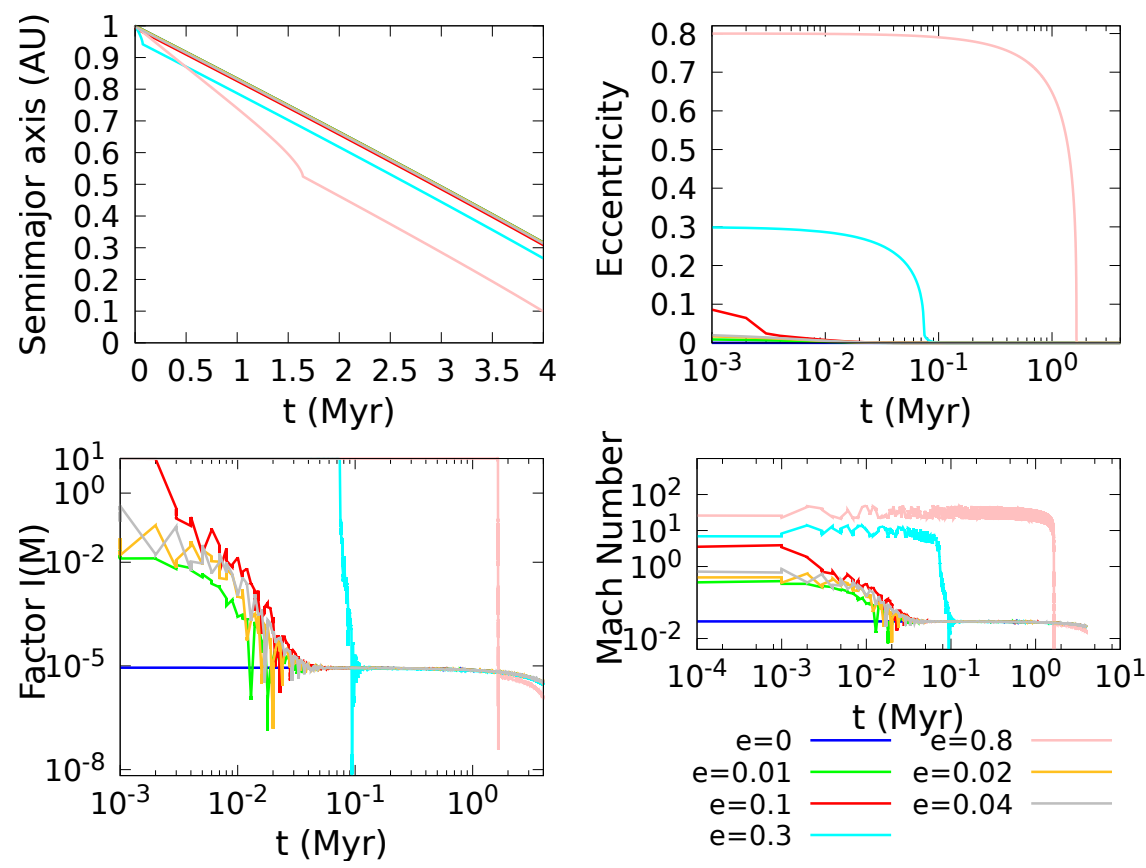

Figure 4. A summary of the effect of the gas dynamical friction force on particle with a mass of $10^{22} \mathrm{~kg}$ with different initial eccentricities. The orbits starts at $1 \mathrm{AU}$ and they are planar orbits. Going clockwise from the top-left the orbital elements are the semi major axis, eccentricity, the Mach number $(M)$ and the factor $I(M)$ ploted in function of the time.

of orbits between two successive outputs using the information of the orbital frequency (mean motion, $n$ ) to obtain an averaged orbital period, which is then slightly adjusted so that the position of the bodies at the end of the 5,000 y timesep matches that recorded in the original simulation. The mean orbital period is then used to calculate the fast variation of the mean longitude within the timestep. Our interpolation deals with events of collisions (merge) and ejection of the proto-planets at the exact moment that these events happen. We eliminate the proto-planets when they are ejected from the Solar System $(e>1.0)$. When a collision occurs, the remaining proto-planet gets a new mass equal to the sum of the masses of the two bodies involved in the event and we put to zero the mass of the proto-planet eliminated during the merge, so that it won't have any further dynamical influence on the planetesimals. The masses of proto-planets and giant planets do not increase due to planetesimal accretion in the simulations. There are other works using interpolation in order to reassess evolution from previous simulations (Deienno et al. (2011); Roig \& Nesvorný (2015); Roig et al. (2016); Deienno et al. (2018); de Sousa et al. (2018) to cite a few).

When we interpolate the evolution of the massive bodies, we rescale their semi-major axes according to the desired location of Jupiter at time $t$, given an imposed migration pattern $a_{\text {jup }}(t)$. In other words, if $a^{I}(t)$ is the semi-major axis of a body $I$ at time $t$ in Izidoro et al. (2015a) output (or the interpolated value from the output) and $a_{j u p}^{I}$ is the semi major axis of Jupiter, we convert $a^{I}$ into $a(t)=a^{I}(t) R(t)$ where $R(t)=a_{\text {jup }}(t) / a_{\text {jup }}^{I}(t)$. All the other orbital elements are kept unchanged. Due to the fact that the direct perturbation of the protoplanets embedded in the planetesimal disc is larger and happens in a shorter timescale than any perturbation by a possible secular or mean 

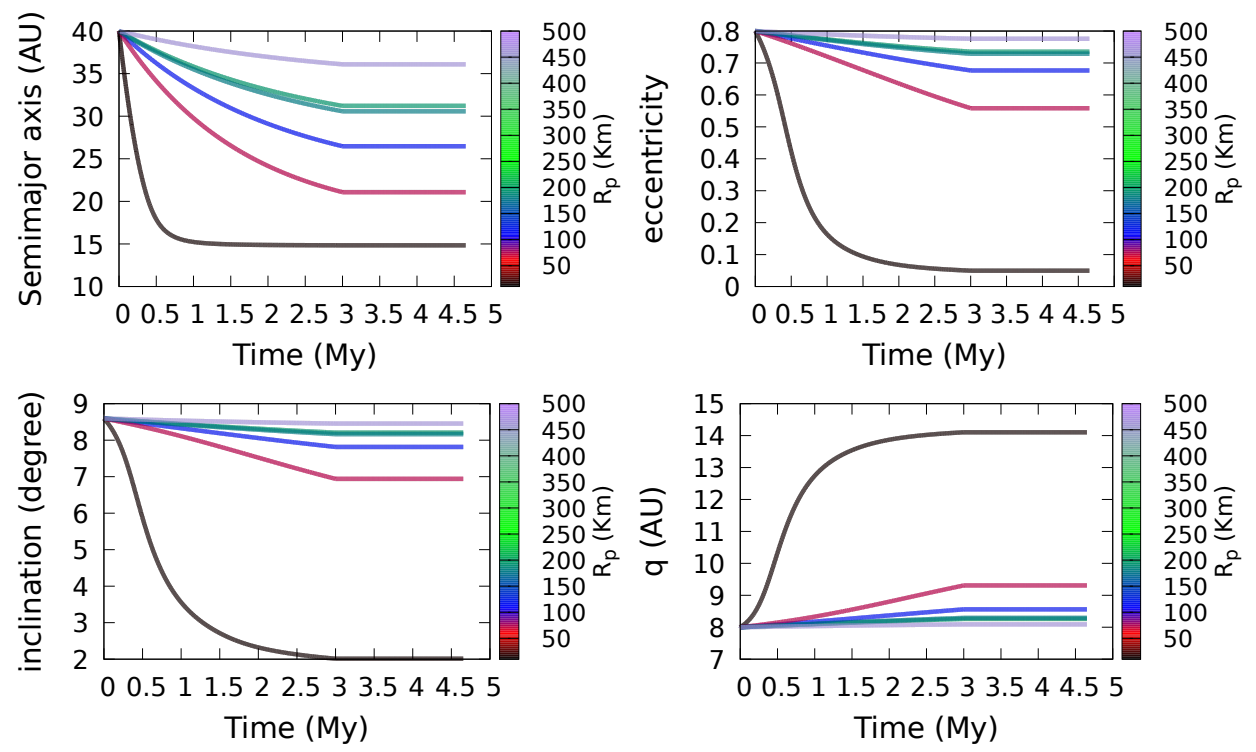

Figure 5. A summary of the action of the aerodynamic gas drag and dynamical friction gas drag on a planetesimal's orbital elements. In this simulation, we used the surface density presented in Fig. 2 assuming a uniform decay over time of $3 \mathrm{My}$, the gas is removed at $3 \mathrm{My}$. Going clockwise from the top-left the orbital elements are the semi major axis, eccentricity, perihelion and inclination ploted in function of the time.

motion resonance, this should not place a problem nor change our main results/conclusions. Then, the orbital elements are converted into positions, which are used in the N-body code to compute the forces that the massive bodies exert on the planetesimals. In order to preserve the orbital periods, the length of the timestep $d t^{I}$ of Izidoro's simulation is rescaled as $d t=d t^{I}\left[a_{\text {jup }}(t) / a_{\text {jup }}^{I}(t)\right]^{3 / 2}$. The simulation time is incremented by $d t$ (and not $d t^{I}$ ). By doing so, the total duration of the simulation increases, if Jupiter is farther than the original 3.5 AU (e.g. the simulation illustrated in Fig. 7 where Jupiter is kept at $5 \mathrm{AU}$ will be $5.12 \mathrm{My}$ instead of the original $3 \mathrm{My}$ ). Concerning the gas, given the rescale factor $R(t)$ on semi-major axes, the surface density at the heliocentric distance $r$ is computed as $\Sigma(r / R(t)) /(R(t))^{2}$, where $\Sigma$ is the surface gas density in Izidoro et al. (2015a)'s simulation. As for the gas velocity in the azimuthal direction, it is rescaled as $v_{\theta}(r / R(t)) / \sqrt{R(t)}$. The rescaled gas quantities are used to compute forces due to gas drag and gas dynamical friction on the planetesimals. The aspect ratio of the disk is $h(r / R(t))$. In hydrodynamical simulations, the migration of the planets temporarily affects the surface density distribution of the gas, for instance by pushing gas inside its orbit and leaving a slightly depleted outer disc behind (acting like a snowplough as shown by Crida \& Bitsch (2017)). However, on longer timescales, the situation stabilises and migration rate of the planets is proportional to the viscosity of the disk and the shape of the gap is not affected when the planet moves (Robert et al. 2018). We show in Fig. 6 how the location of the surface density of the gas disk changes overtime during the Jupiter migration.

\subsection{Results}

In this section, we present the results of our numerical simulations considering a planetesimal disk interacting gravitationally with the Sun, with the giant planets and the proto-planets (described in section 2.2.2), and with the gas using the protoplanetary disk described in section 2.2.1. The goal is 


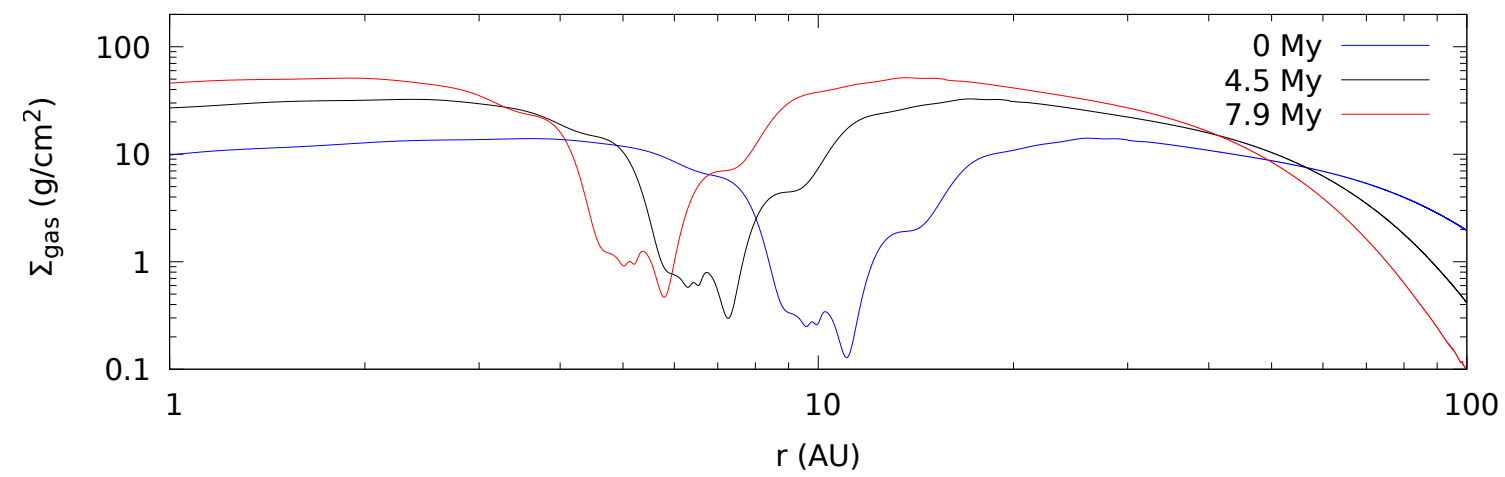

Figure 6. The surface density profiles $\Sigma(r / R) / R^{2}$. Different colours lines correspond to different times $(0$ My, 4.5 My and 7.9 My). It corresponds the case with Jupiter migrating from 10 to 5 AU.

to determine the dynamical state of the planetesimal disk after the gas phase, in particular to assess the distance between Neptune and the inner edge of the planetesimal disk.

We performed five simulations using the package REBOUND code (Rein \& Liu 2012) with the integrator IAS15 (Rein \& Spiegel 2015). Each simulation represents a different imposed orbital migration history of Jupiter, with all other massive bodies rescaled accordingly from the Izidoro et al. (2015a) simulation, as explained before. The semimajor axis of Jupiter evolved as a function

$$
a_{J}(t)=a_{J}^{\prime}+a^{\prime} \exp (-t / \lambda)
$$

where $a_{J}^{\prime}$ is the final semimajor axis of Jupiter and $a^{\prime}$ is set so that $a_{J}^{\prime}+a^{\prime}$ is the initial position of Jupiter. We adopted an e-folding timescale $\lambda=1 \mathrm{My}$ for all the simulations.

Table 1 shows our set of the simulations. We performed a simulation considering the giant planets on non-migrating orbits (section 2.3.1), with Jupiter and Saturn at 5 and 6.8 AU, and a simulation considering Jupiter and Saturn migrating outward (section 2.3.2) in agreement with Grand Track Scenario (Walsh et al. 2011; Brasser et al. 2016). However, because the migration history of Jupiter and Saturn in their natal gas disk is poorly constrained (Bitsch et al. 2015), we also performed simulations in which Jupiter migrated inward from 10 to $5 \mathrm{AU}$, from 15 to $5 \mathrm{AU}$ and from 20 to $5 \mathrm{AU}$ (in section 2.3.3). In all these scenarios, Saturn migrated inward as well because we rescaled Saturn's semi-major axis using Jupiter's semimajor axis (as we have shown in Section 2.2).

For each imposed migration history, we used four independent simulations using different planetesimals sizes, with diameters of $1 \mathrm{~km}, 10 \mathrm{~km}, 100 \mathrm{~km}$ and $1000 \mathrm{~km}$. The planetesimal size matters because of the size-dependent effects of gas drag and gas dynamical friction. All the simulations start with fully formed Jupiter and Saturn, multiple planetary embryos and a planetesimal disk with 4,000 planetesimals with eccentricities and inclination of 0.001 and extended from 10 up to 60 AU. 
Table 1. Set of simulations

\begin{tabular}{ccc}
\hline \hline Name of the simulation set & Criteria for Jupiter's migration & Time of the dispersal of the gas \\
\hline Jup_static & Jupiter on non-migrating orbits at 5 AU & $5.2 M y r$ \\
Jup_outward & Jupiter migrating outward from 2 to 5 AU & $3.7 M y r$ \\
Jup_10AU_in & Jupiter migrating inward from 10 to $5 \mathrm{AU}$ & $7.9 M y r$ \\
Jup_15AU_in & Jupiter migrating inward from 15 to 5 AU & $11 M y r$ \\
Jup_20AU_in & Jupiter migrating inward from 20 to 5 AU & $14.9 M y r$ \\
\hline
\end{tabular}



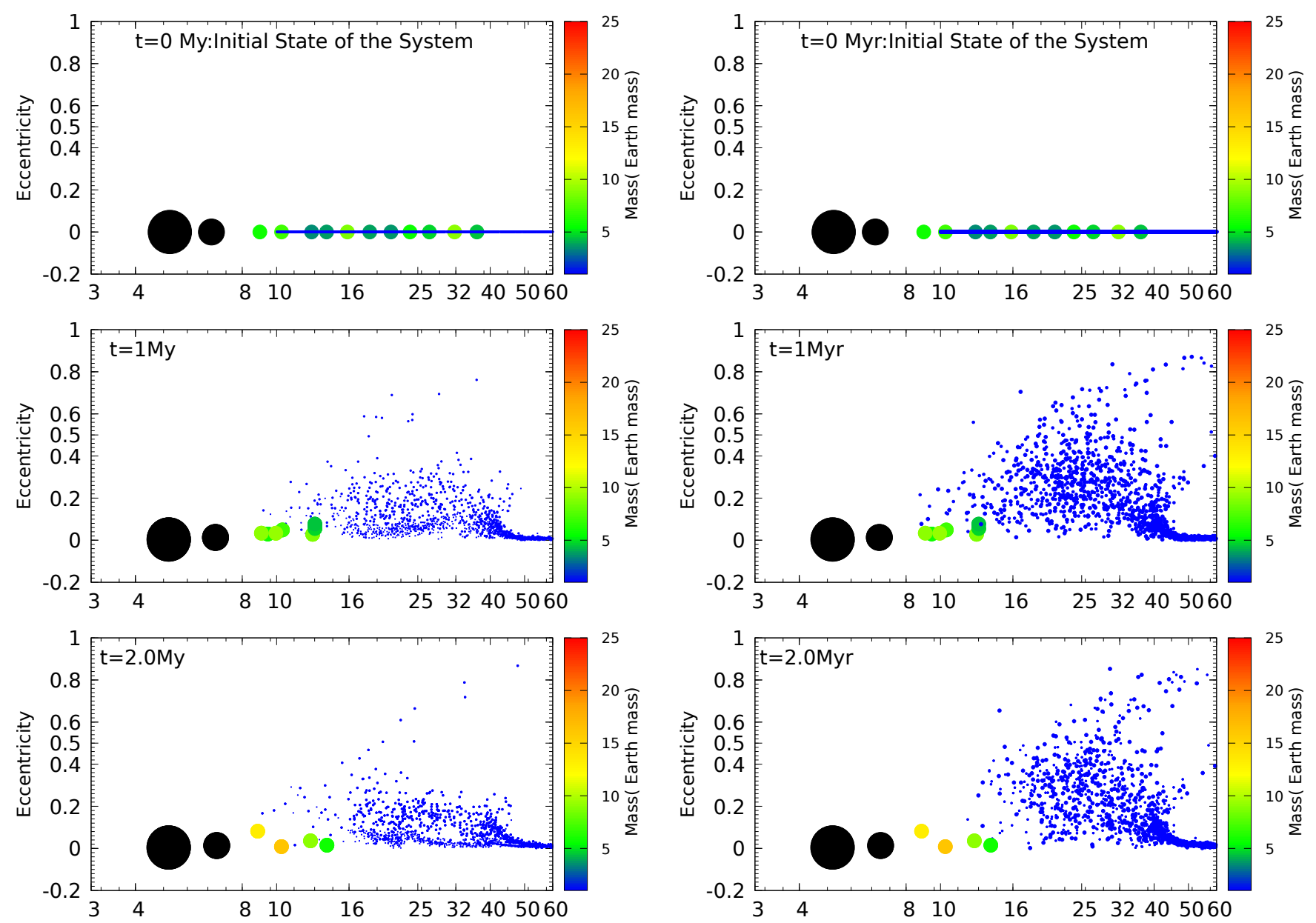

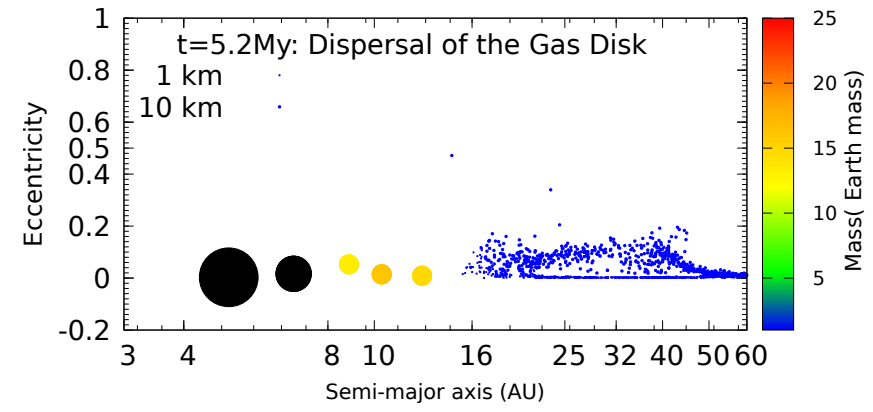

(a)

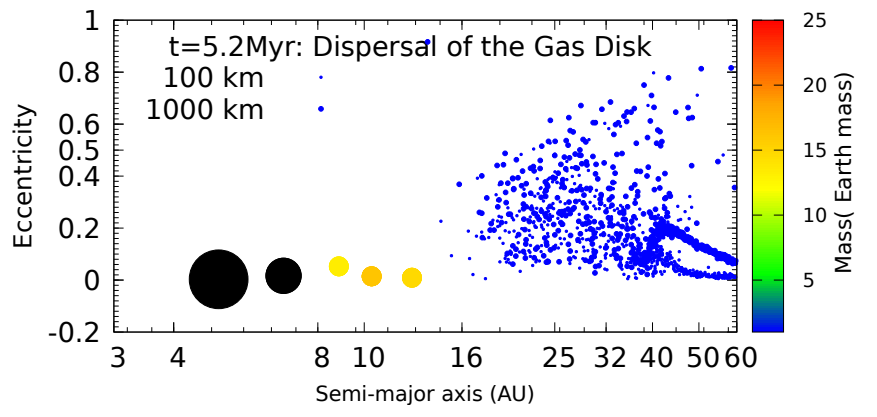

(b)

Figure 7. Eccentricity/Semi-major axis plot portraying the dynamical evolution of the case Jup_static (table 1). Panel (a) represents the dynamical evolution for a co-addition of planetesimals with sizes of $1 \mathrm{~km}$ and $10 \mathrm{~km}$. Panel (b) shows the dynamical evolution but for a co-addition of planetesimals sizes of $100 \mathrm{~km}$ and $1000 \mathrm{~km}$. The color box represents the mass of the particles, except for Jupiter and Saturn (we use the size of each point to represent the mass of Jupiter and Saturn and the planetesimals sizes).

\subsubsection{Jupiter on non-migrating orbits}

Figures 7 and 8 show the eccentricity, inclination and semi-major axis evolution for the simulation set Jup_static. The gas lifetime (hence the duration of the simulation) in this simulations is set to 

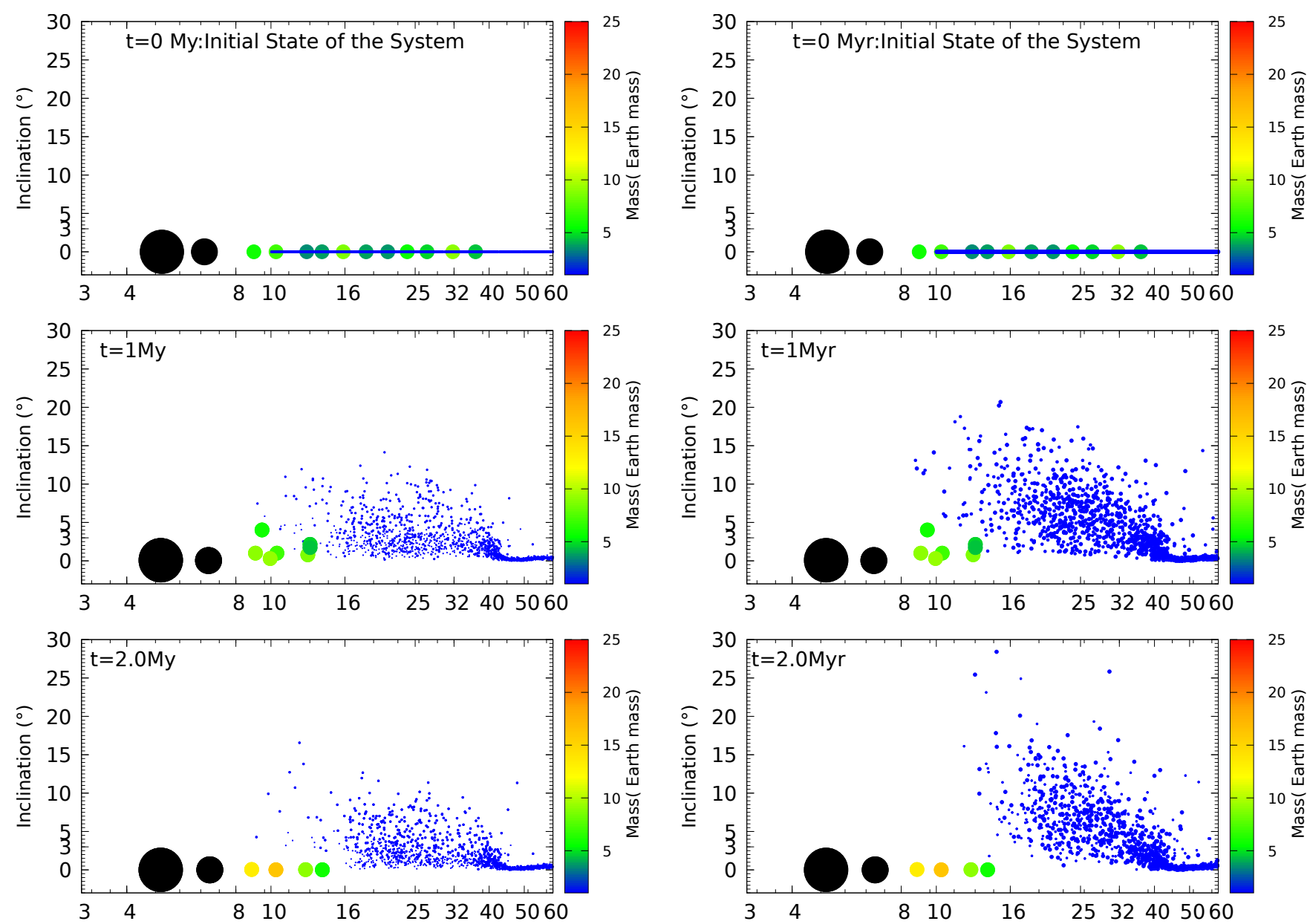

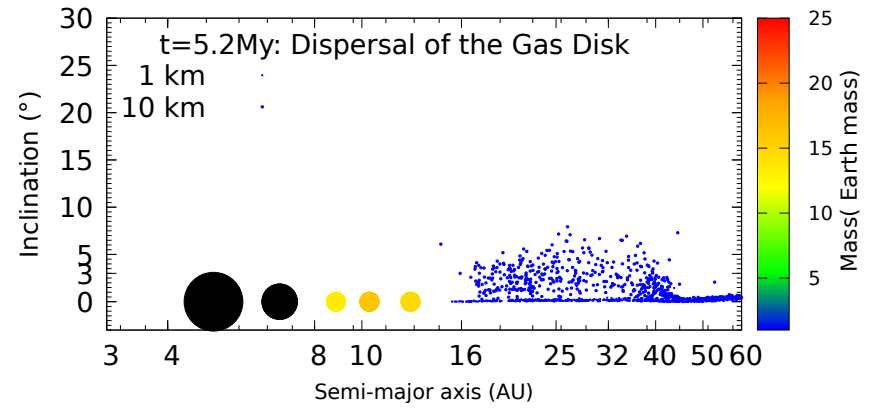

(a)

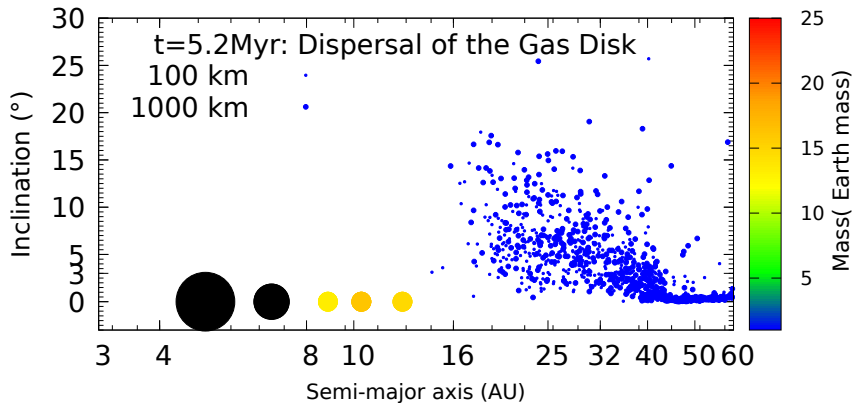

(b)

Figure 8. Inclination/Semi-major axis plot portraying the dynamical evolution of the case Jup_static (table 1). Panel (a) represents the dynamical evolution for a co-addition of planetesimals with sizes of $1 \mathrm{~km}$ and $10 \mathrm{~km}$. Panel (b) shows the dynamical evolution but for a co-addition of planetesimals sizes of $100 \mathrm{~km}$ and $1000 \mathrm{~km}$. The color box represents the mass of the particles, except for Jupiter and Saturn (we use the size of each point to represent the mass of Jupiter and Saturn and the planetesimals sizes).

5.12 Mys. Notice that the smaller are the planetesimals, the colder is the final disk. This is because of the stronger effect of the aerodynamic gas drag and a negligible gas dynamical friction (see in Fig. 5). During the dynamical evolution of the planetary embryos and the giant planets showed in Fig. 1 
and described in the section 2.1, the planetesimal disk is depleted by close encounters and collisions with the planetary embryos or Jupiter and Saturn.

In the end of our simulation we defined the inner edge of the planetesimal disk in semi major axis $\left(a_{\text {edge}}\right)$ and perihelion distance $\left(q_{\text {edge }}\right)$ such that their cumulative normalized $a$ and $q$ distributions have values of $N\left(a<a_{\text {edge }}\right)=0.05$ and $N\left(q<q_{\text {edge }}\right)=0.05$ respectively (rather arbitrary but it is done so that rogue planetesimals with small $a$ or $q$ do not define the inner disk's edge; in other words, we accept that $5 \%$ of the planetesimals are outliers, inwards of the defined disk's edge) (observe in Fig. 9 (a) and (b)). Neptune is defined as the outer most planet and at the end of the simulation it has semi-major axis of $12.58 \mathrm{AU}$ and perihelion of $12.46 \mathrm{AU}$.

We see that the inner edge of the disk in the end is quite close to Neptune's orbit and is planetesimal size dependent. Although the proto-planets were initially distributed up to $\sim 40$ AU, they migrated out of the 20-40 AU region rather quickly. Therefore they could dynamically excite the planetesimal population in that region, but not deplete it significantly. Moreover, gas drag partially damped the planetesimals' eccentricities and inclinations once the proto-planets left their natal region. Thus the final separation in semi-major axis between Neptune and the disk is 5.09 AU for the km-size planetesimals and $8.16 \mathrm{AU}$ for the $1,000 \mathrm{~km}$-size planetesimals. The separation is smaller for small planetesimals because gas-drag tends to circularize the excited objects near their perihelion distance. If considered in $q$ (perihelion distance) space, however, the separation shrinks to $5.07 \mathrm{AU}$ and 0.55 AU respectively. The big difference between $a_{e d g e}$ and $q_{\text {edge }}$ for the 1,000 km-planetesimals is due to the non-zero eccentricity of the latter.

The radial distance (measured with semimajor axes) between Neptune and the planetesimal disk is less important when the planetesimals are on eccentric orbits and have lower perihelion distances. In this case, Neptune is in closer contact with the planetesimal disk. Deienno et al. (2017) showed that even though there is a resolution dependence between the distance of Neptune and inner edge of the disk, the distance in the range that we found here would lead to an instability before $400 \mathrm{Myr}$, and Quarles \& Kaib (2019) found even earlier instability times. We foresee that such a small separation will lead to an early instability, as we will test in section 3.

\subsubsection{Jupiter migrating outward}

Figures 10 and 11 show the eccentricity, inclination and semi-major axis evolution for the simulation set Jup_outward. In this simulation, Jupiter is migrating outward from 2 to 5 AU and the simulation begins with a planetesimal disk extended from 10 to $60 \mathrm{AU}$ (blue points). The gas lifetime is 3.7 Myr. The separation between Neptune and the inner edge of the planetesimal disk is even smaller than before. In fact, this separation is $2.63 \mathrm{AU}$ for the km-size planetesimals and $6.91 \mathrm{AU}$ for the $1,000 \mathrm{~km}$-size planetesimals in semi-major axis (Fig. 12 (a)). The separation in pericenter is 1.99 $\mathrm{AU}$ for the $\mathrm{km}$-size planetesimals and $0.46 \mathrm{AU}$ for the 1,000 km-size planetesimals (Fig. 12 (b)). This is due to the fact that the planets moved towards the planetesimal disk and the average distance from the growing planets was larger than the average distance in the previous simulation. Another important effect in this simulation is the resonant shepherding, which transports planetesimals along with migrating planets in a size-dependent way (e.g. Fogg \& Nelson 2005; Raymond et al. 2006). 


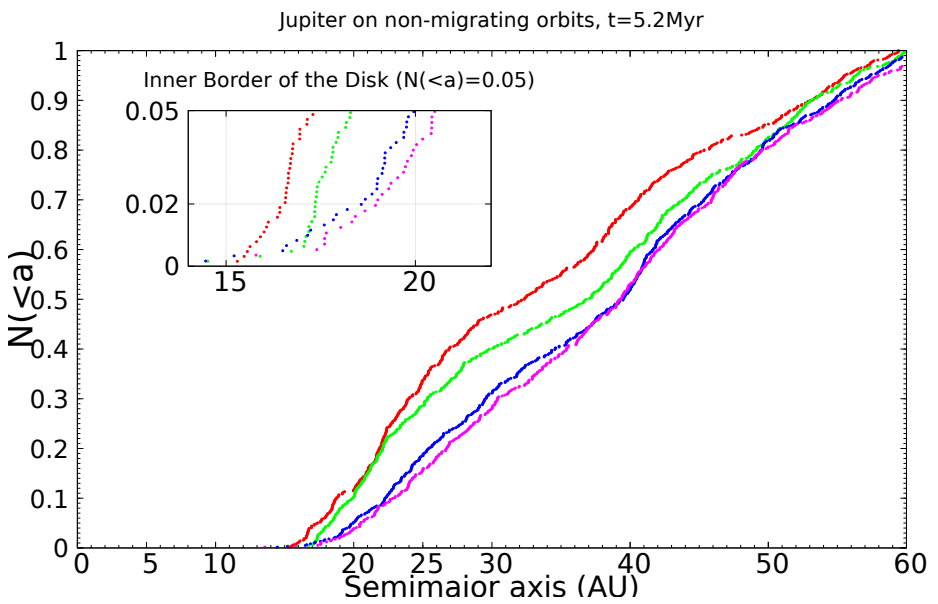

(a)

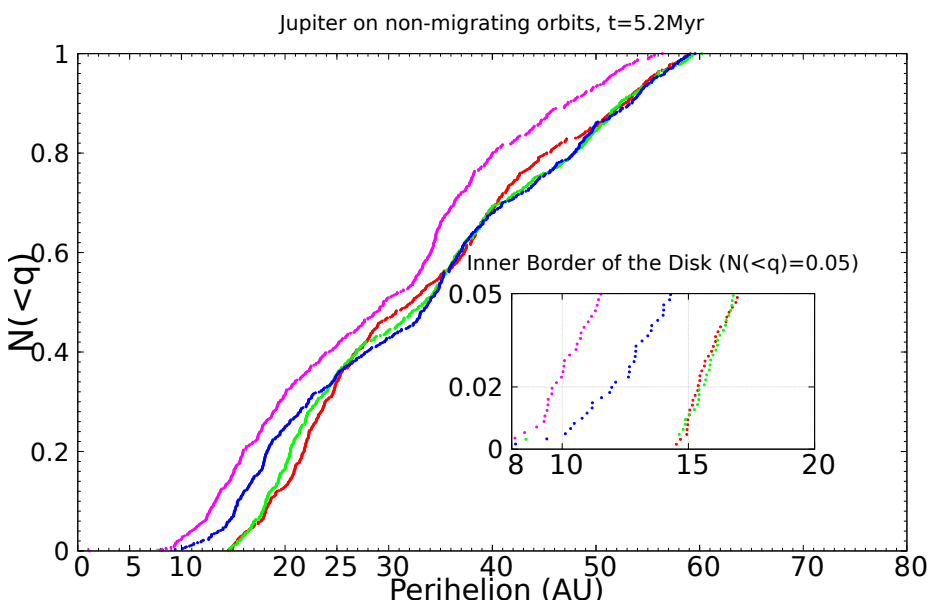

(b)

Figure 9. Cumulative normalized semi-major axis (panel (a)) and perihelion (panel (b)) distributions of the planetesimal disk at the dispersal of the gas in the simulation set Jup_static (table 1). The red, green, blue and magenta curve colors represent the cumulative distributions for different planetesimal's sizes, 1 $\mathrm{km}, 10 \mathrm{~km}, 100 \mathrm{~km}, 1000 \mathrm{~km}$ respectively. We defined the border of the planetesimal disk in the end of our simulation as the value such their cumulative normalized distributions have values of $N(<a)=0.05$ and $N(<q)=0.05$ (smaller panels (a) and (b)). We used these cumulative distributions to calculate the distance between the inner border of the disk and Neptune. Note: Neptune is defined as the outer most ice giant planet with semi-major axis of $12.58 \mathrm{AU}$ and perihelion of $12.46 \mathrm{AU}$. 

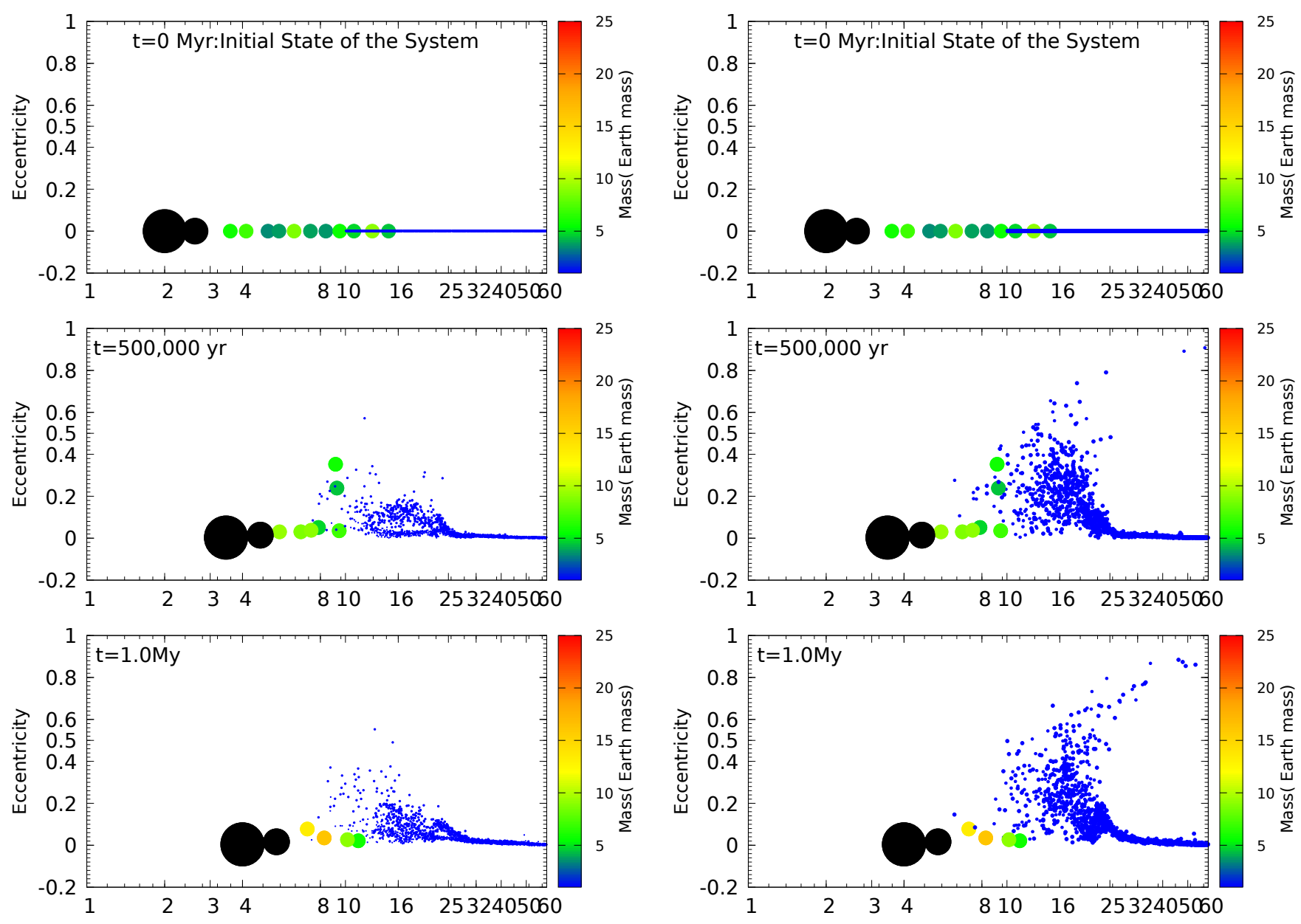

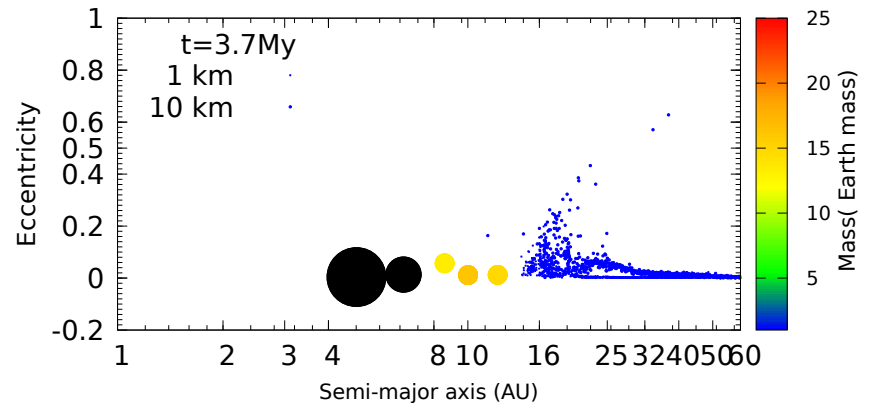

(a)

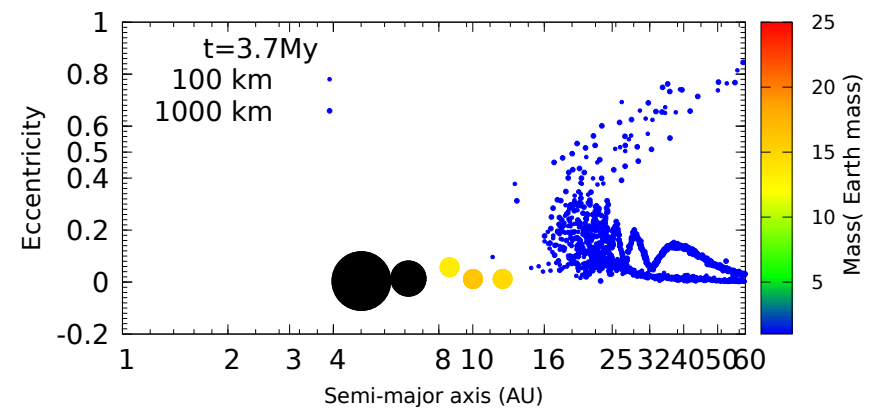

(b)

Figure 10. The same as Fig. 7, but for the simulation set Jup_outward (table 1) where Jupiter is assumed to migrate from 2 to $5 \mathrm{AU}$. The total duration of the simulation is $3.7 \mathrm{My}$. 

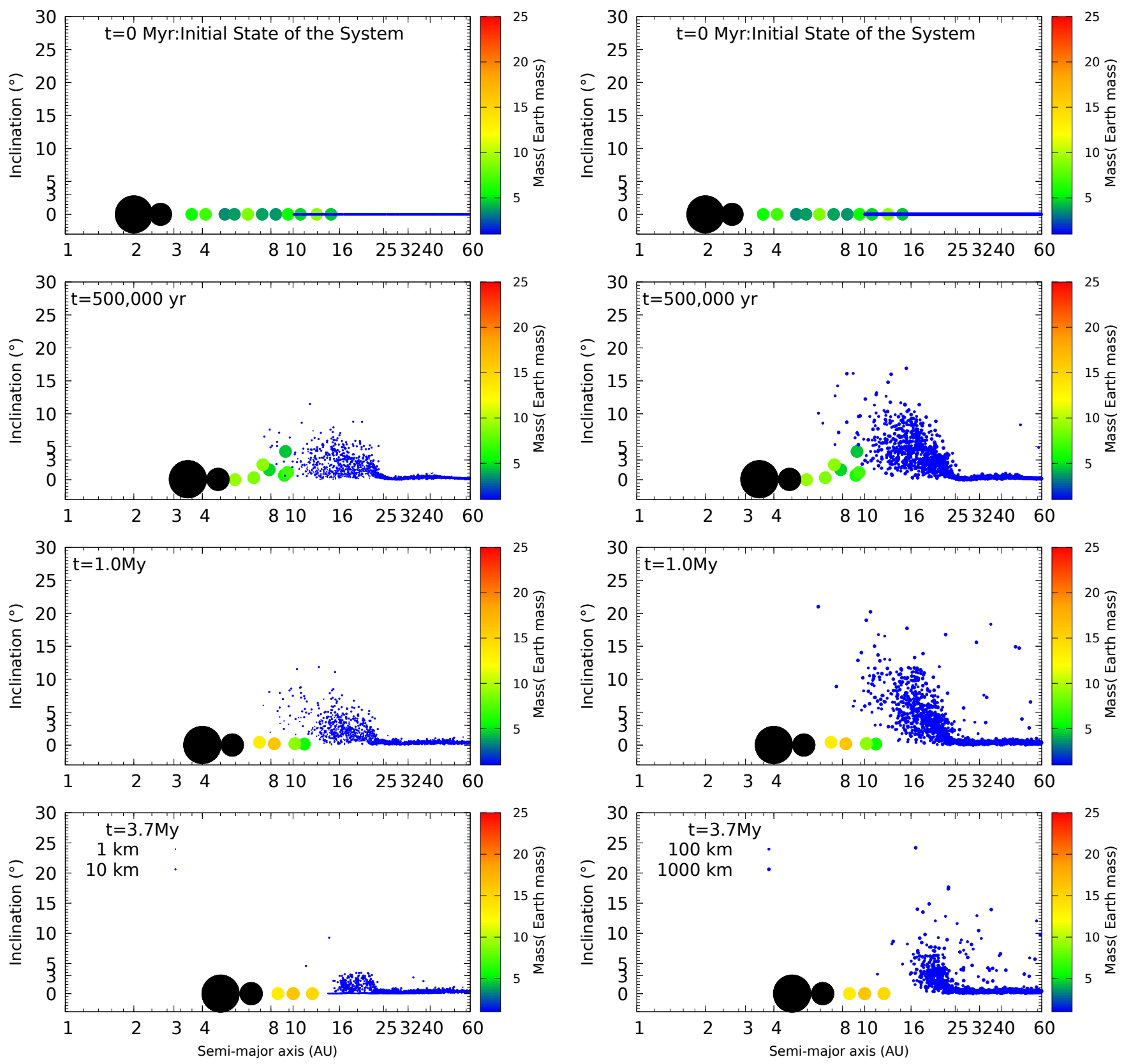

(a)

(b)

Figure 11. The same as Fig. 8, but for the simulation set Jup_outward (table 1) where Jupiter is assumed to migrate from 2 to $5 \mathrm{AU}$. The total duration of the simulation is $3.7 \mathrm{My}$. 


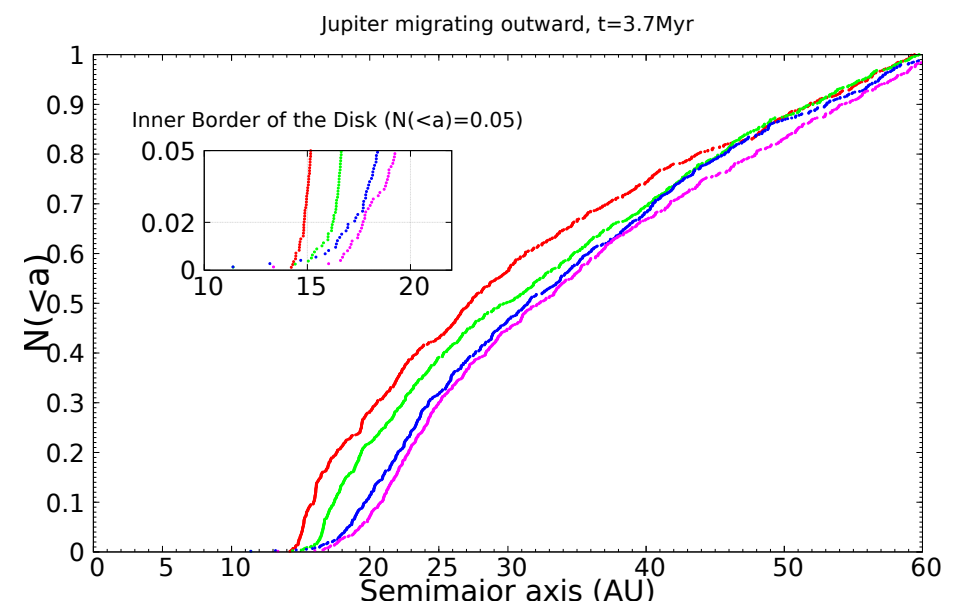

(a)

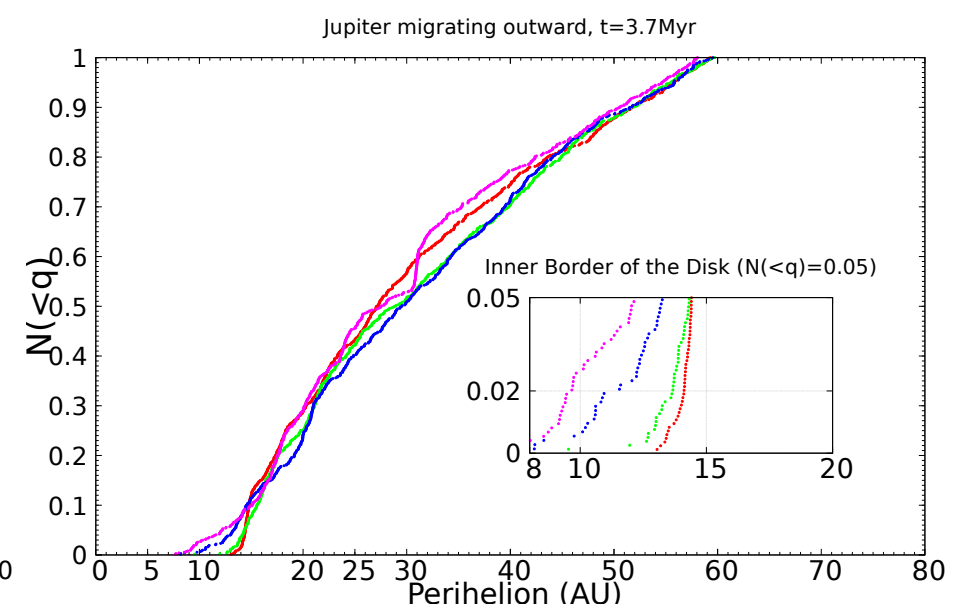

(b)

Figure 12. The same as Fig. 9, but for the simulation set Jup_outward (table 1). 


\subsubsection{Jupiter migrating inward}

The cases of inward migration of Jupiter, Jup_10AU_in, Jup_15AU_in and Jup_20AU_in, are presented in Figures 13, 14, 15, 16, 17, 18, 19, 20 and 21, respectively. The simulations begin with a planetesimal disk extended from 20 to $60 \mathrm{AU}$ (blue points). We see that the wider is the migration range of Jupiter, the larger is the final gap between the position of Neptune and the inner edge of the disk. For instance, for 1,000 km-size planetesimals, the separation in $q$ increases from $0.66 \mathrm{AU}$, for a 5 AU inward migration (Case Jup_10AU_in, see Fig. 15), to 11.95 AU for a 15 AU inward migration (Case Jup_20AU_in, see Fig. 21). The reason is that Saturn and the proto-planets starting farther out in the disk can remove more efficiently the 20 - 30 AU population of planetesimals by scattering or accretion-concerning to planetesimal's accretion, the masses of the planets and proto-planets do not increase due to planetesimal accretion thus, we just removed the planetesimais which accreted with these objects. Therefore, for large inward migration of Jupiter there may be the possibility that the planet instability occurs after a long time. We will check this in section 3. Note however that, if Jupiter migrated from 10 AU or beyond, the final disk's inclination excitation beyond 40 AU for 100 $\mathrm{km}$ and larger objects exceeds significantly that ones observed in the cold Kuiper belt population, as discussed next. 

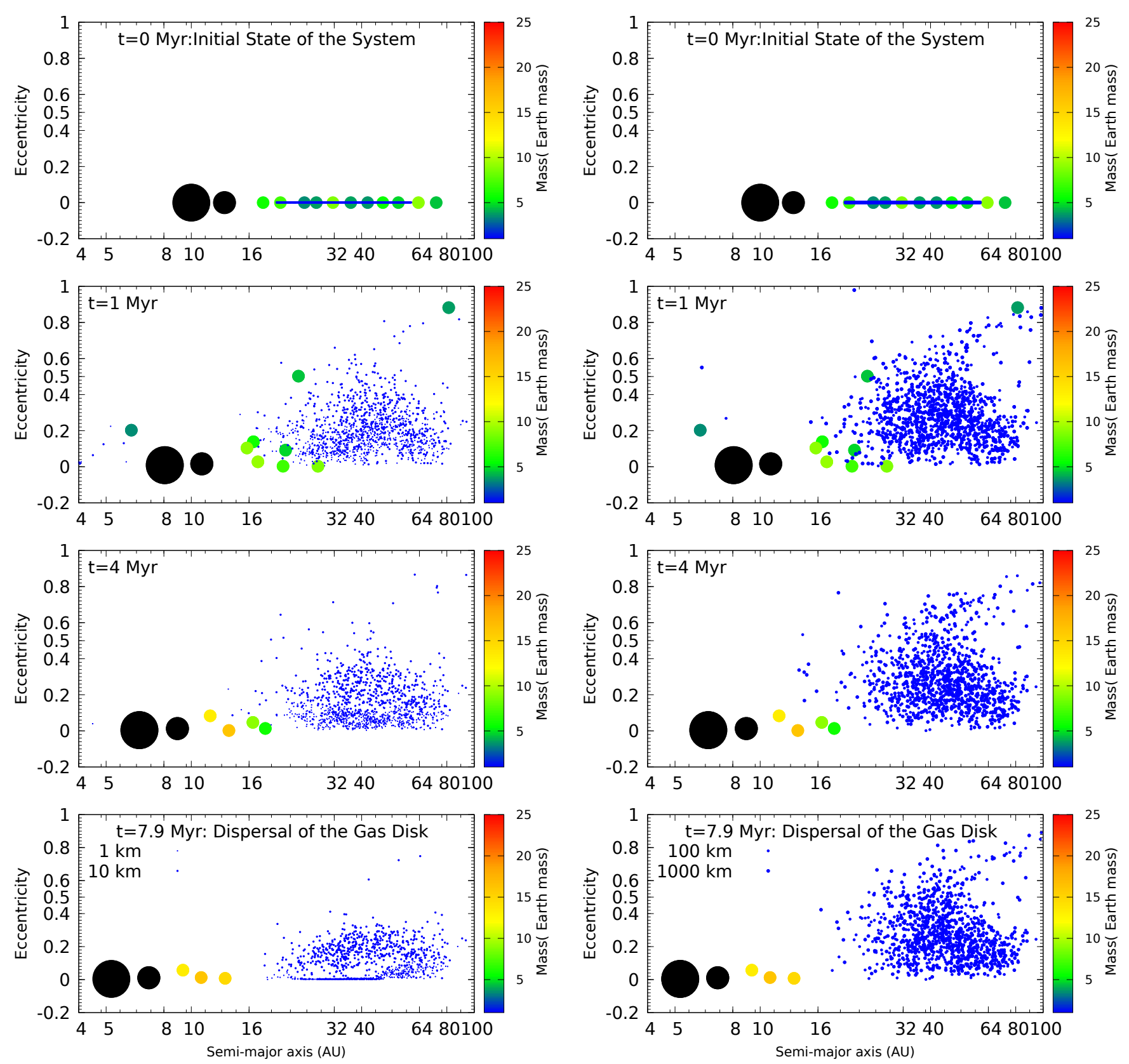

(a)

(a)

Figure 13. The same as Fig. 7, but for the simulation set Jup_10AU_in (table 1) where Jupiter is assumed to migrate from 10 to $5 \mathrm{AU}$. The total duration of the simulation is $7.9 \mathrm{My}$. 

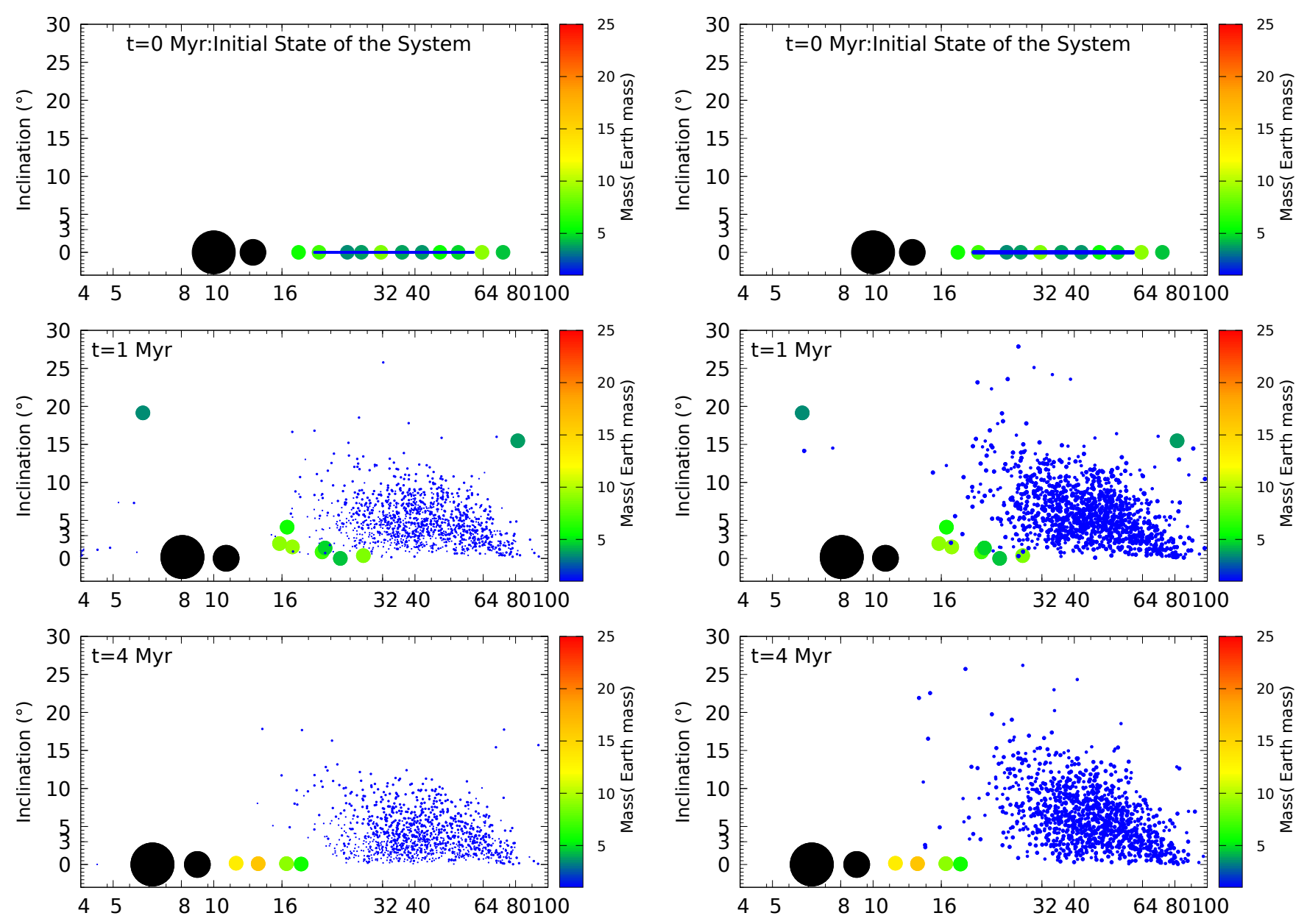

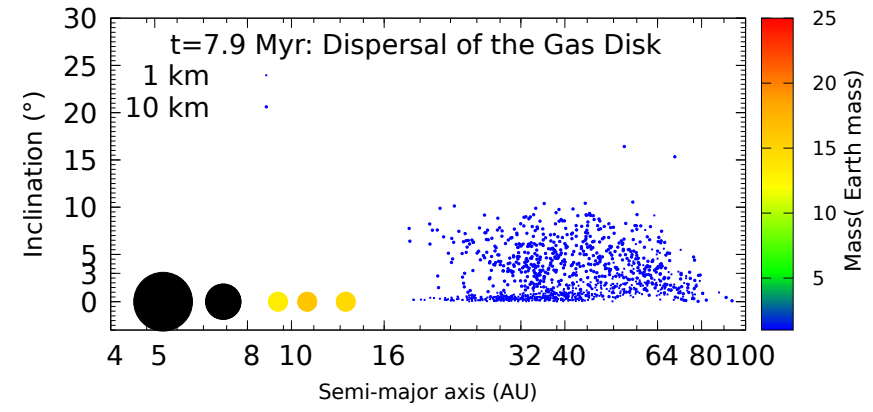

(a)

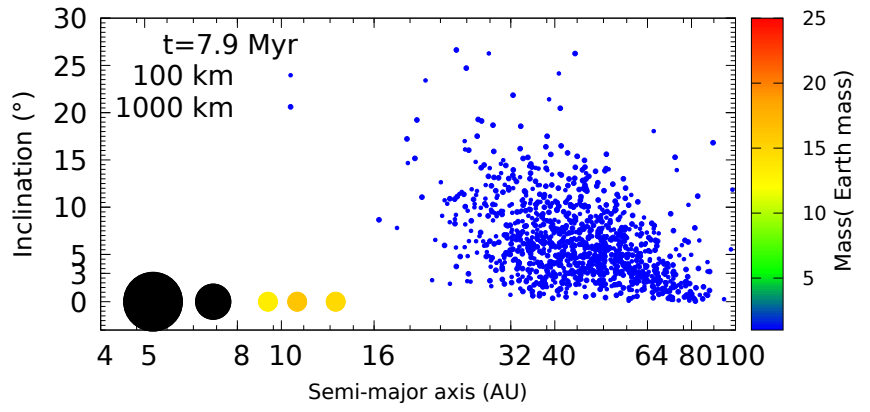

(a)

Figure 14. The same as Fig. 8, but for the simulation set Jup_10AU_in (table 1) where Jupiter is assumed to migrate from 10 to $5 \mathrm{AU}$. The total duration of the simulation is $7.9 \mathrm{My}$. 


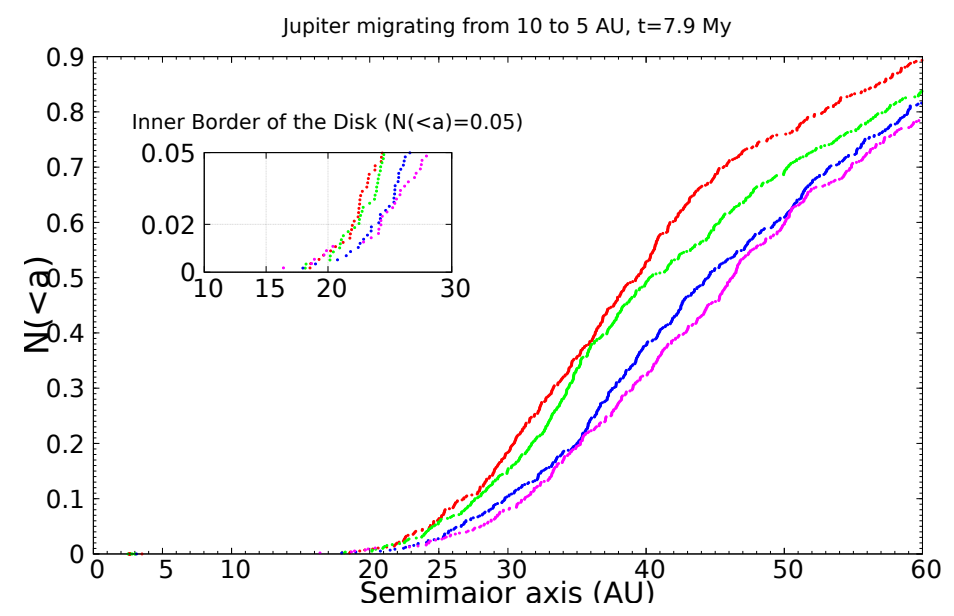

(a)

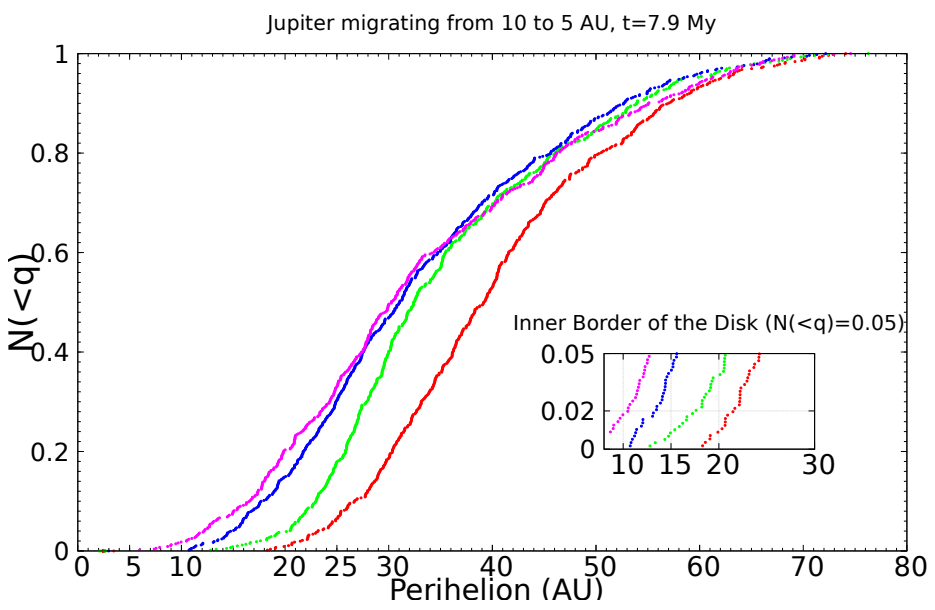

(b)

Figure 15. The same as Fig. 9, but for the simulation set Jup_10AU_in (table 1). 

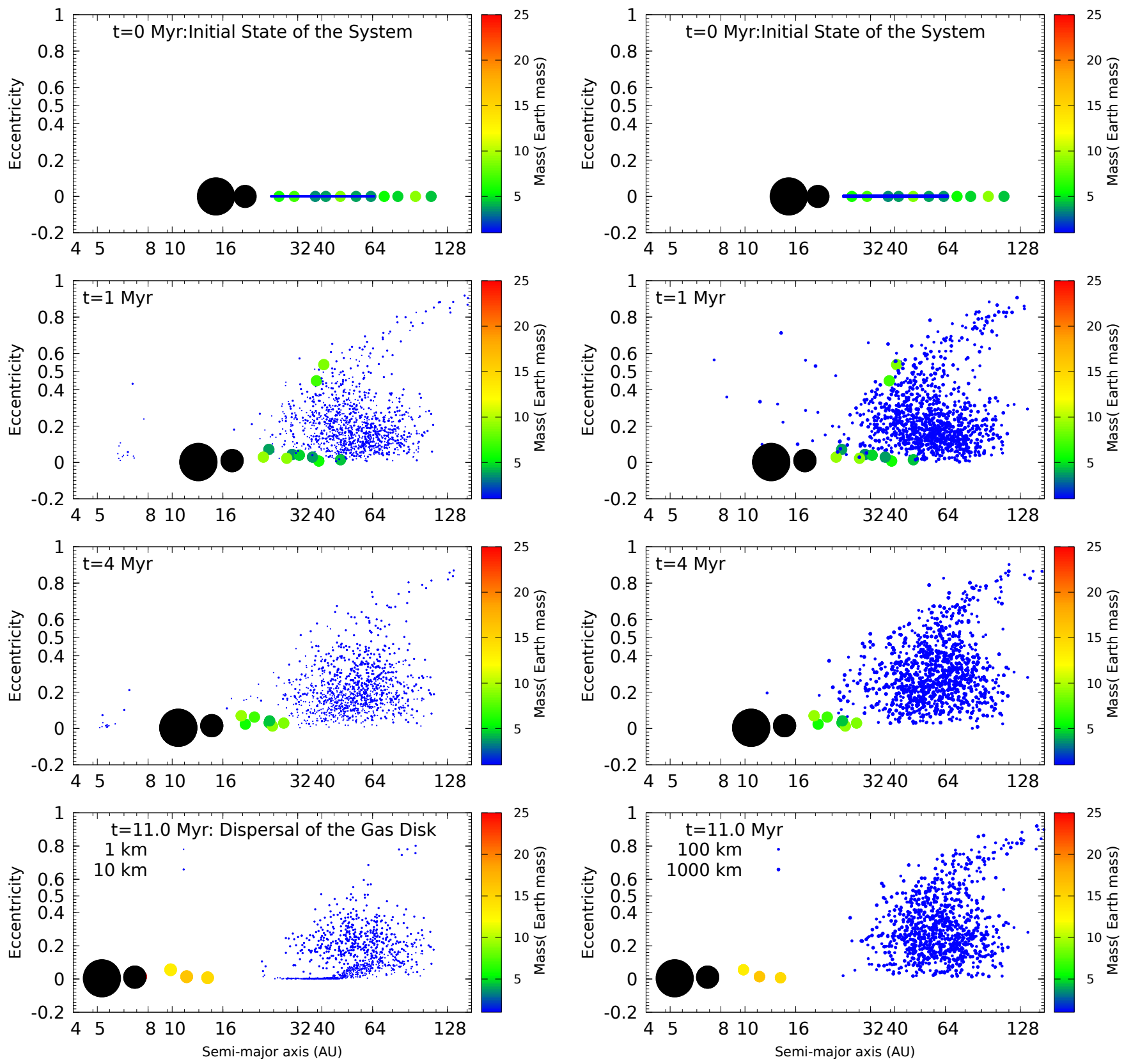

(a)

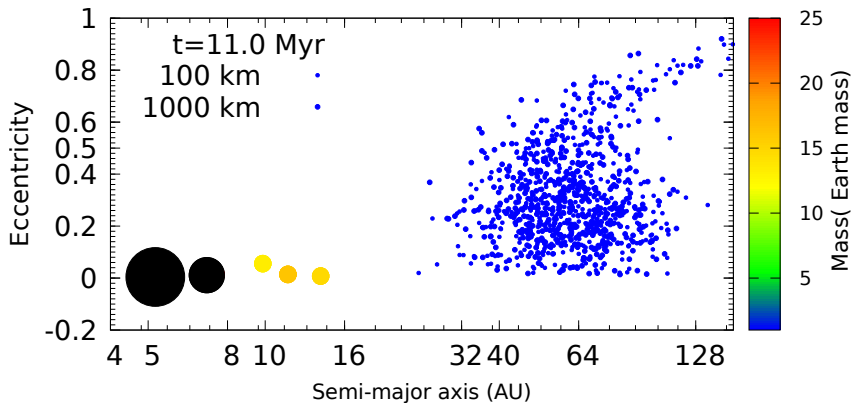

(a)

Figure 16. The same as Fig. 7, but for the simulation set Jup_15AU_in (table 1) where Jupiter is assumed to migrate from 15 to $5 \mathrm{AU}$. The total duration of the simulation is $11 \mathrm{My}$. 

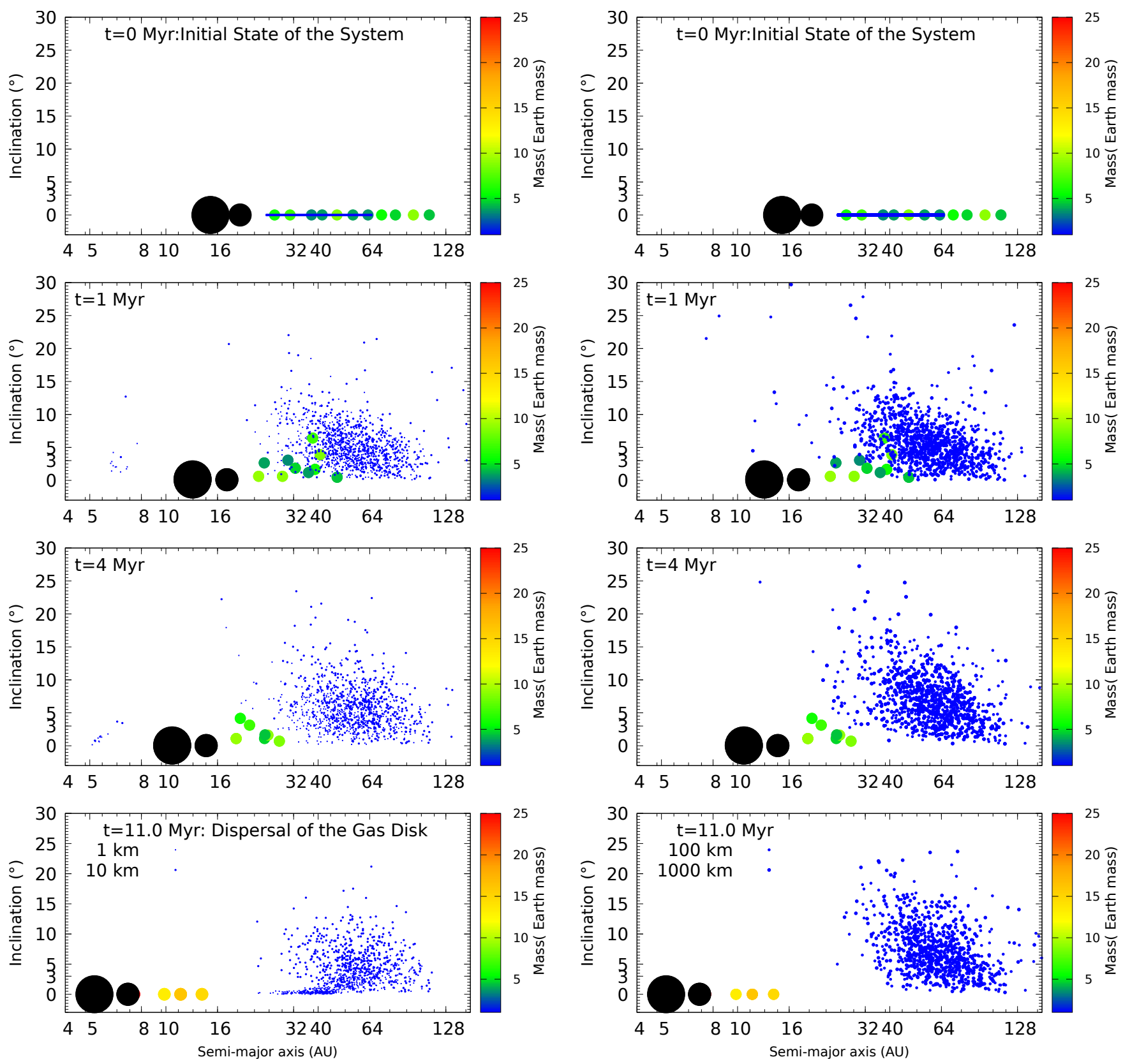

(a)

(a)

Figure 17. The same as Fig. 8, but for the simulation set Jup_15AU_in (table 1) where Jupiter is assumed to migrate from 15 to $5 \mathrm{AU}$. The total duration of the simulation is $11 \mathrm{My}$. 


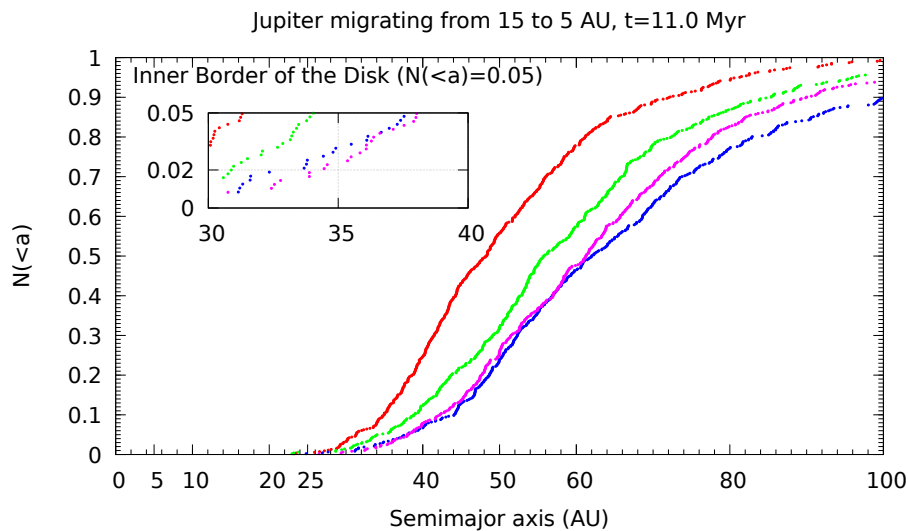

(a)

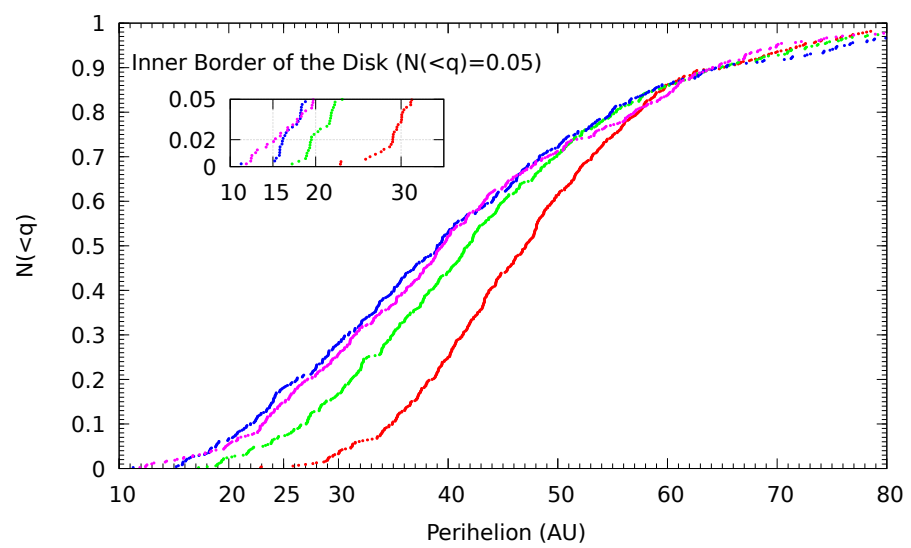

(b)

Figure 18. The same as Fig. 9, but for the simulation set Jup_15AU_in (table 1). 

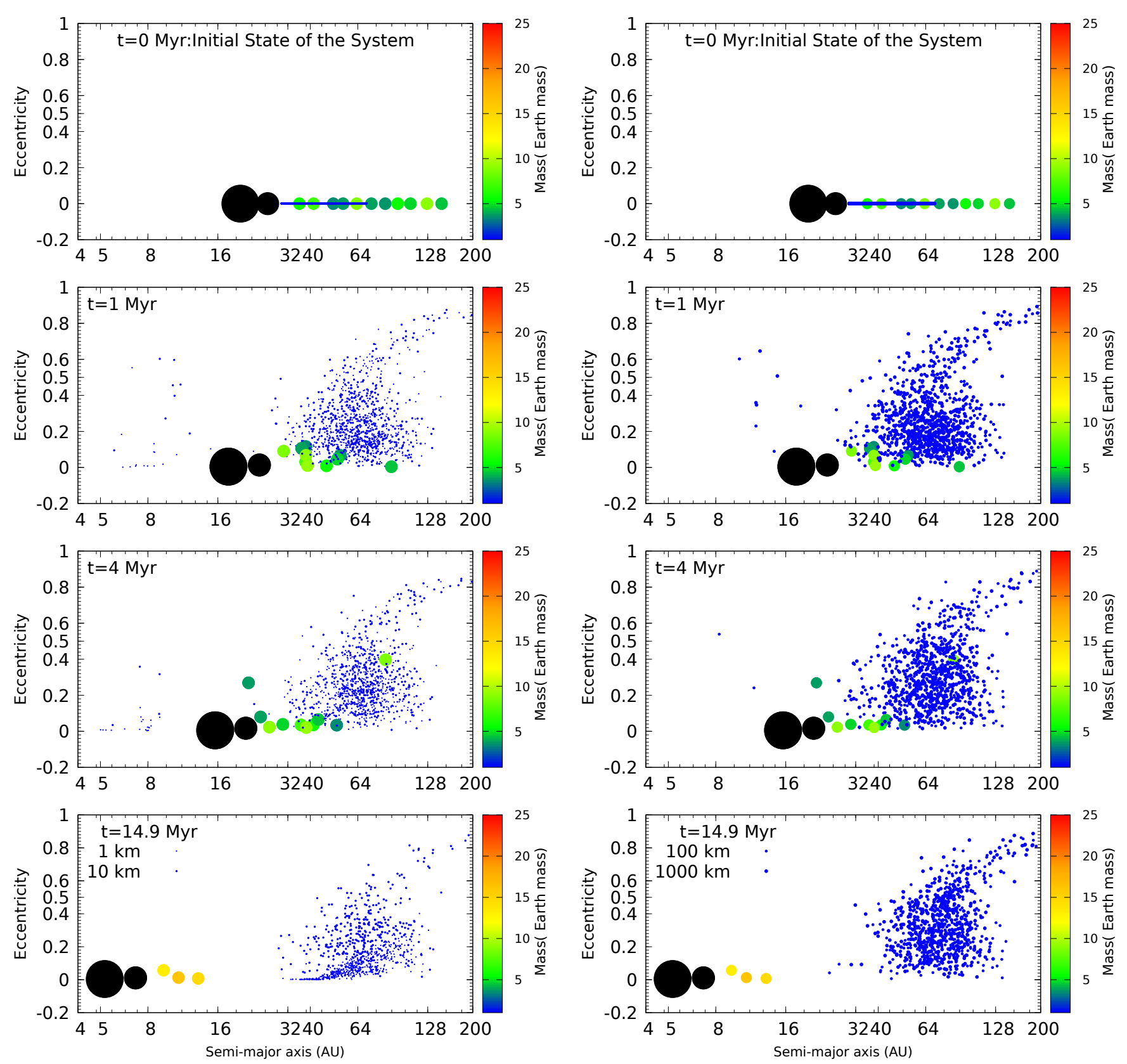

(a)

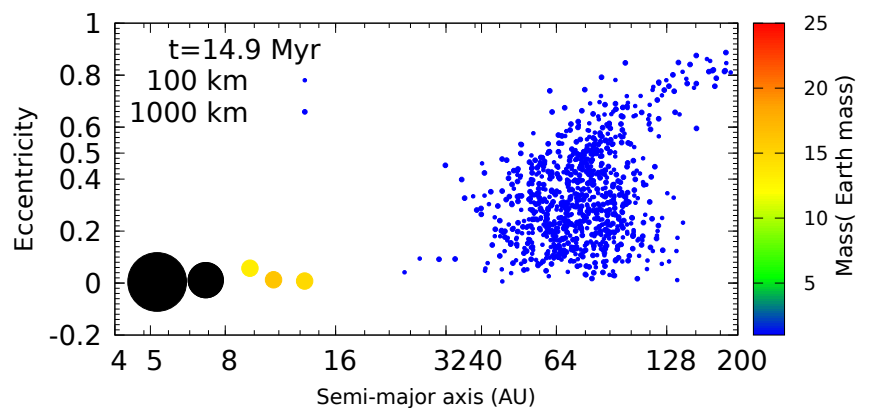

(b)

Figure 19. The same as Fig. 7, but for the simulation set Jup_20AU_in (table 1) where Jupiter is assumed to migrate from 20 to $5 \mathrm{AU}$. The total duration of the simulation is $14.9 \mathrm{My}$. 

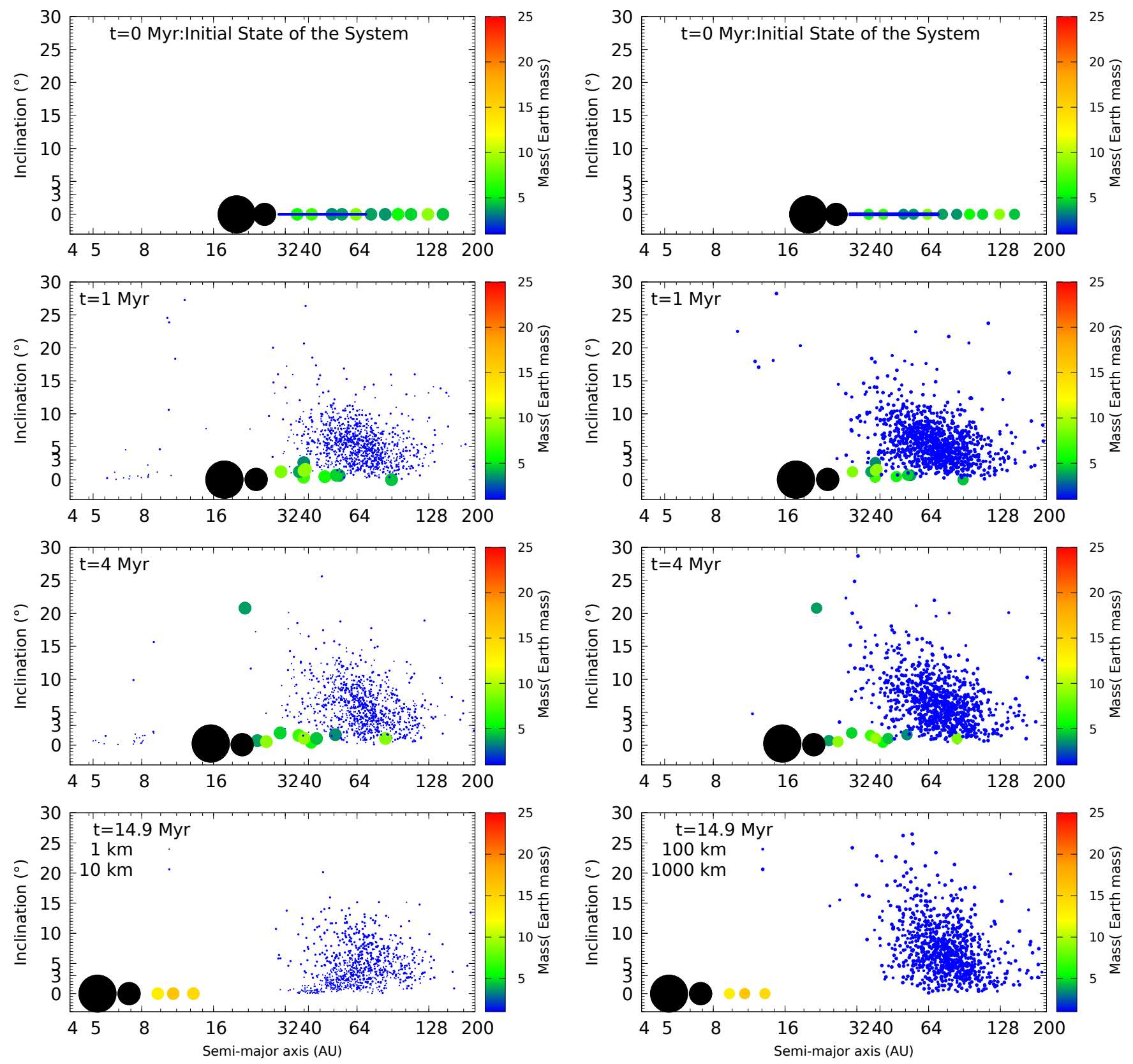

(a)

(b)

Figure 20. The same as Fig.8, but for the simulation set Jup_20AU_in (table 1) where Jupiter is assumed to migrate from 20 to $5 \mathrm{AU}$. The total duration of the simulation is $14.9 \mathrm{My}$. 


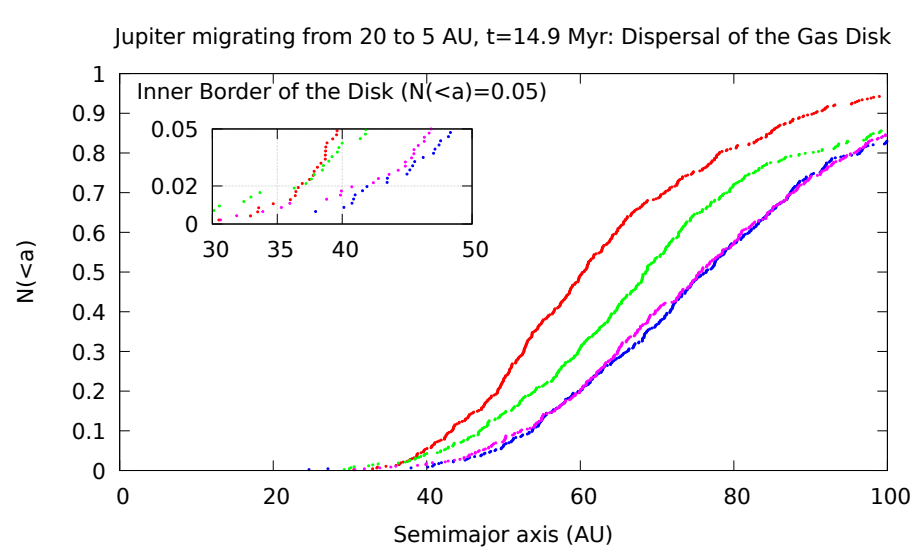

(a)

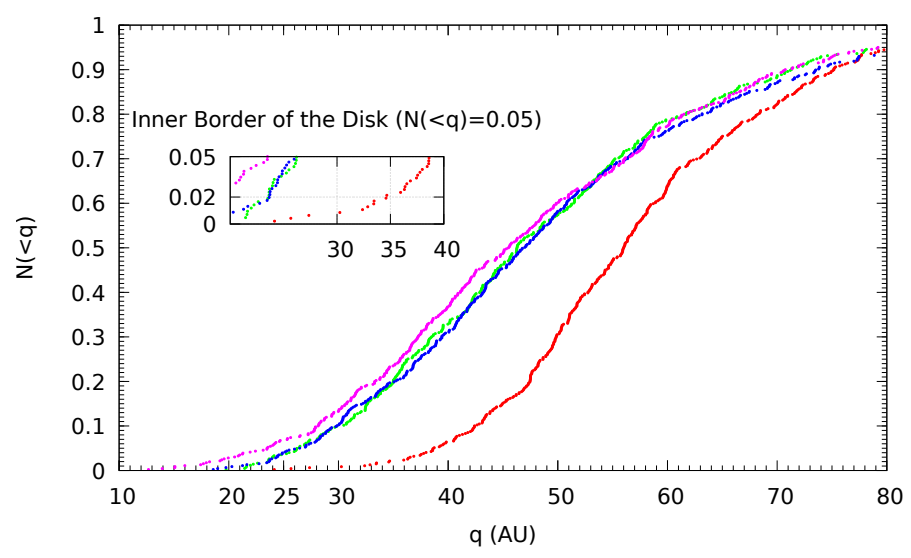

(b)

Figure 21. The same as Fig. 9, but for the simulation set Jup_20AU_in (table 1). 


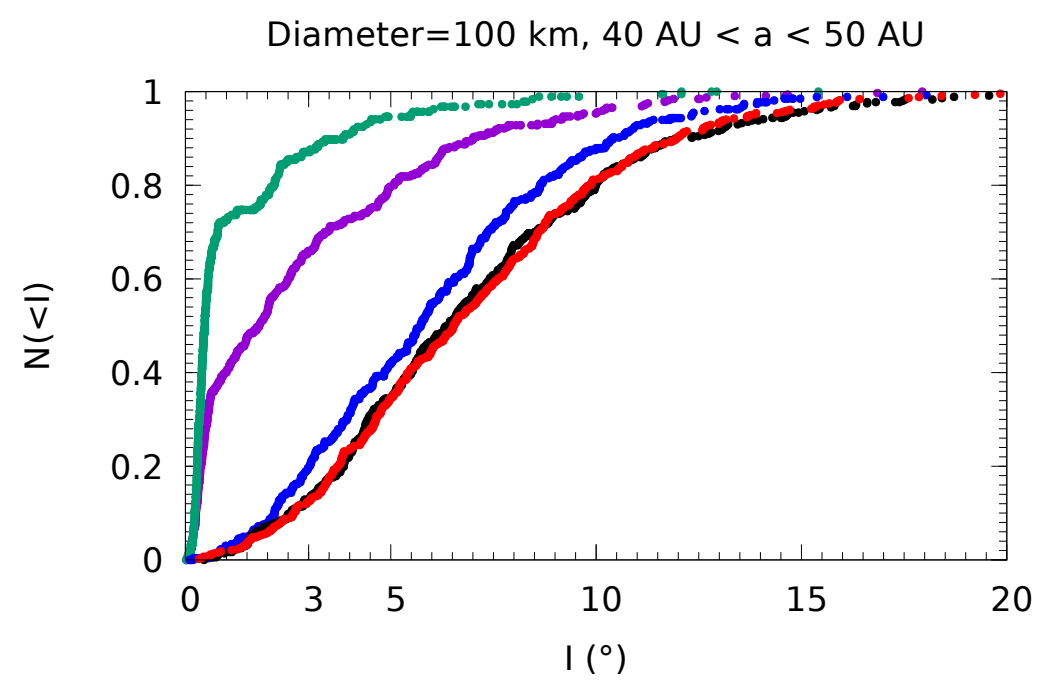

Figure 22. Cumulative normalized inclination distribution $(N(<I))$ for $100 \mathrm{~km}$ objects presented in the local cold population $40-50$ AU at the end of our five simulations (Jup_static (purple), Jup_outward (green), Jup_10AU_in (blue), Jup_15AU_in (black) and Jup_20AU_in (red), table 1). Note the cases with Jupiter migration from 10 or beyond the local 40 - 50 AU population have a final inclination dispersion of 10 degrees. Whereas, the others cases have a final inclination dispersion smaller than 5 degrees.

\subsection{Which planetesimal disks are consistent with the primordial Kuiper belt?}

The cold Kuiper belt population is very low in eccentricity and inclination and is notoriously confined with 4 degrees from the invariant plane. We can use this as a constraint on our planetesimal disks, as there is no clear mechanism to damp planetesimals' orbits after the dissipation of the gaseous disk. Figure 22 shows the cumulative inclination distributions for $100 \mathrm{~km}$ planetesimals in all of our planetesimal disks. Planetesimal disks in which Jupiter migrated from 10 AU or beyond tend to leave the local 40 - 50 AU population too excited to be compatible with the cold Kuiper Belt. For instance, in the simulation with Jupiter migrating from $10 \mathrm{AU}$, the final inclination dispersion in the $40-50 \mathrm{AU}$ region is 10 degrees (for $100 \mathrm{~km}$ objects) ( blue curve in Fig. 22). This is because the planetary embryos precursors of Uranus and Neptune are located beyond Jupiter and Saturn and therefore, if Jupiter is originally beyond 10 AU, some of the planetary embryos are initially resident in the Kuiper Belt region. The embryos migrate away from their original location, but in doing so they excite the local planetesimal population. Gas drag damping is weak for objects of $100 \mathrm{~km}$ in size or larger, and therefore this excitation remains until the gas disk fade out, in contrast with small inclination excitation observed for the cold Kuiper Belt population. We notice that the simulations with Jupiter on a fixed orbit, or migrating outward, give an inclination excitation in the $40-50$ AU region (green and purple curves in Fig. 22) that does not exceed the excitation of the cold Kuiper Belt.

\section{CONSTRAINING THE TIMING OF THE GIANT PLANET INSTABILITY}

We now address the timing of the giant planet instability. For statistical reasons, we make use of all simulations from Izidoro et al. (2015a) that produced good matches to the outer Solar System, 
meaning that they formed at least two roughly equal-mass ice giants. This constitutes a large enough sample that we can make statistical arguments. We first test the stability of the giant planet systems alone to test for self-triggered instabilities (§3.1). Next we test the stability of the same systems while including our self-consistently generated planetesimal disks (§3.2).

Here we define time zero as the end of the gaseous disk phase, i.e. the end of the simulations presented in Section 2.3. We measure the time of the giant planet instability relative to this. The instability is defined as the beginning of the close-encounter phase among the planets. It happens when the giant planets break their resonant chain configuration.

\subsection{Self-triggered Instability}

Izidoro et al. (2015a) never tested whether their final systems of gas giants and ice giants remained stable after the gas disk phase. Many of such systems become unstable very quickly. This happens when the planets, in particular the ice giants, are too eccentric for long-term stability or in a too compact resonant chain. Indeed, it is well-known that resonant chains may go unstable (e.g. Matsumoto et al. 2012; Izidoro et al. 2017). We statistically studied the stability of 27 giant planet systems from Izidoro et al. (2015a). Each of these systems contained at least two roughly equal-mass ice giants similar to Uranus and Neptune, and many contained one or more additional surviving small planets (ice giant-mass or less). For each of these systems we scaled the semi major axis of all planets to place Jupiter at $5 \mathrm{AU}$ (see section 2.2.2). Then we integrated each system for up to 1 Gyr to determine whether the system remained stable or underwent a self-triggered instability.

Our initial conditions were the orbital elements of the planets at the end-time of the gas phase from Izidoro et al. (2015a). Since instabilities are so sensitive to initial conditions, we performed 10 simulations of each system to generate a distribution of outcomes. For each simulation we did slight changes on the planet initial conditions: a random phase chose in the interval $(-0.003,0.003)$ degrees is added to each orbital angles of the planets, including the mutual inclinations. These changes are small enough not to modify the stability conditions of the original planetary systems. Indeed, all closens of stable systems remain stable (in absence of planetesimal perturbations). We integrated each system for 1 Gyr using a time step of 0.5 years.

Fig. 23 shows the outcome of these simulations. Some systems go unstable quickly and others remain stable for 1 Gyr. Just under half (48\%) systems remain stable for 1 billion of years. The vast majority $(80 \%)$ of unstable systems had instabilities within $10 \mathrm{Myr}$. The reason why many of Izidoro et al. (2015a)'s planetary systems are self-unstable compared to the systems built as initial conditions of the Nice model (Morbidelli et al. 2007) is that in Izidoro et al. (2015a)'s case (which we used in this work) the planetary systems is continuously unstable until Uranus and Neptune are built and at that point there is not much gas to damp the orbits of the planets, weheras Morbidelli et al. (2007) considered 4 full formed planets that were captured in mean motion resonance in sequence.

The presence of an outer planetesimal disk may have a significant effect on the stability of a system of giant planets, in particular when the masses of the outermost planets are comparable to the disk mass (Raymond et al. 2010). Thus, in the next section (§3.2) we test how the stability of giant planet systems changes when we include the planetesimal disks that we found in section 2.3.

\subsection{Planetesimal disk-triggered Instability}

We now perform simulations to determine the timing of a planet instability triggered by the interaction with the planetesimal disk, making use of the planetesimal disks produced by Section 2.3. 


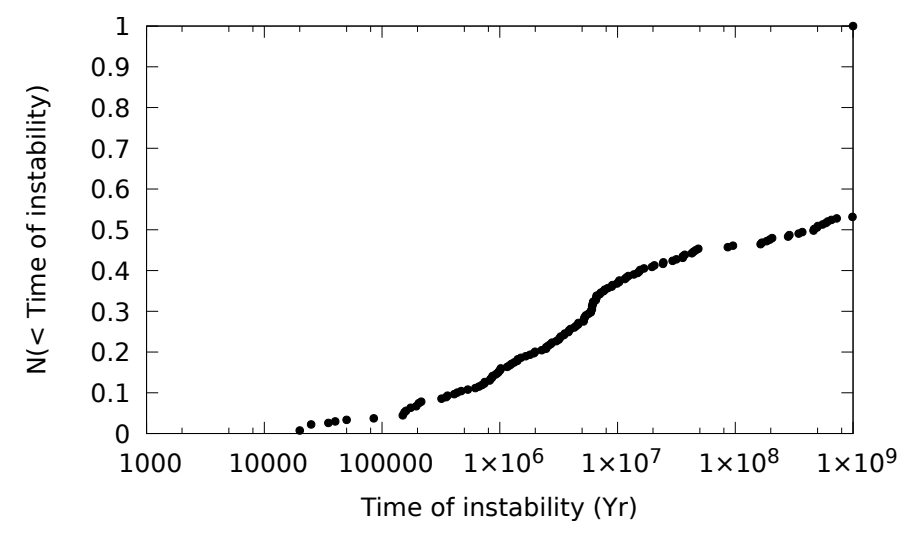

Figure 23. Panel shows the cumulative normalized distribution of the time of instability calculated during our simulations after the gas dispersal and without any planetesimal disk.

The simulations presented in the Section 2.3 treated planetesimals as test particles. We adopted 4 sizes for the computation of the gas-drag effects and hence the final orbital distributions. Now, if we want to investigate the effects of the planetesimal disk onto the planets, we need to combine the orbital distribution of these 4 categories of particles and assign a mass to them. We explain how we do this in the subsection 3.2.1 below.

\subsubsection{Mass of the Planetesimal disks}

The total mass of the primordial planetesimal disk is expected to be 20 Earth's mass (Nesvorný \& Morbidelli 2012; Nesvorný \& Vokrouhlický 2016). Its original size distribution was reconstructed by Nesvorný \& Vokrouhlický (2016) using many constraints. The first constraint is that Neptune's migration should have been grainy to explain why the fraction of the Kuiper Belt population in resonances is relatively small. This grainy migration requires close encounters of Neptune with massive Pluto-class planetesimals. Therefore, Nesvorný \& Vokrouhlický (2016) argued that the planetesimal disk contained $1000-4000$ Pluto-size objects. For the intermediate sizes $(10<D<$ $500 \mathrm{~km}$ ) Nesvorný \& Vokrouhlický (2016) adopted the Fraser et al. (2014) size distribution from observations of the Kuiper Belt and Jupiter Trojans. This distribution shows a knee at $D \sim 100 \mathrm{~km}$. Other constraints come from the comet's size distribution and the requirement that the overall mass of the disk is finite. In summary, Nesvorný \& Vokrouhlický (2016) model the cumulative distribution $N(>D)$ as a piece-wise power law:

$$
N(>D) \propto D^{-q}
$$

with $q=1$ for $D>500 \mathrm{~km}, q=5$ for $100<D<500 \mathrm{~km}, q=2$ for $10<D<100 \mathrm{~km}, q=3$ for $1<D<10 \mathrm{~km}$ and $q=2$ for $D<1 \mathrm{~km}$. We consider that $N(>1000 \mathrm{~km}), N(>500 \mathrm{~km})$, $N(>100 \mathrm{~km}), N(>10 \mathrm{~km}), N(>1 \mathrm{~km})$ and $N(>0.1 \mathrm{~km})$ are the number of objects with diameter larger than $1000 \mathrm{~km}, 500 \mathrm{~km}, 100 \mathrm{~km}$ and $0.1 \mathrm{~km}$, respectively. The number of objects larger than $500 \mathrm{~km}(N(>500 \mathrm{~km}))$ is a constant to be determined by the total mass of the Planetesimal disk. Thus, we used the cumulative distribution (Eq. 9) to relate the number of objects larger than a size 
$\mathrm{D}(N>D)$ with the respective exponent $q$ :

$$
\begin{gathered}
N(>500 \mathrm{~km})=N(>1000 \mathrm{~km})\left(\frac{1000 \mathrm{~km}}{500 \mathrm{~km}}\right)^{q}, q=1 \\
N(>100 \mathrm{~km})=N(>500 \mathrm{~km})\left(\frac{500 \mathrm{~km}}{100 \mathrm{~km}}\right)^{q}, q=5 \\
N(>10 \mathrm{~km})=N(>100 \mathrm{~km})\left(\frac{100 \mathrm{~km}}{10 \mathrm{~km}}\right)^{q}, q=2, \\
N(>1 \mathrm{~km})=N(>10 \mathrm{~km})\left(\frac{10 \mathrm{~km}}{1 \mathrm{~km}}\right)^{q}, q=3 \\
N(>0.1 \mathrm{~km})=N(>1 \mathrm{~km})\left(\frac{1 \mathrm{~km}}{0.1 \mathrm{~km}}\right)^{q}, q=2 .
\end{gathered}
$$

We used Equations 10 to 14 to calculate the proportional constant $(\gamma)$ of the differential equation $d N(>D)$ defined as:

$$
d N(>D)=\gamma D^{-q-1} d D
$$

We can find $\gamma$ for each region of diameters larger than $D_{1}$ and $D_{2}$, calculating the integral:

$$
N\left(>D_{1}\right)-N\left(>D_{2}\right)=\int_{D_{1}}^{D_{2}} \gamma D^{-q-1} d D
$$

We considered that $N_{0}$ represents the objects larger than $500 \mathrm{~km}$. Thus,

$$
N_{0}=N(>500 k m)=\int_{500}^{+\infty} \gamma_{0} D^{-q-1} d D, q=1
$$

Solving the integral (Eq. 17) in function of $\gamma_{0}$, we write:

$$
\gamma_{0}=500 N_{0}
$$

We can calculate the other proportionality constants, be $\gamma_{1}$ the proportionality constant of the region between 100 and $500 \mathrm{~km}$, with $q=5$ :

$$
N(>100)-N(>500)=\int_{100}^{500} \gamma_{1} D^{-q-1} d D
$$

We can calculate the quantity of mass $\left(M_{D 1, D 2}\right)$ between two different sizes $D_{1}$ e $D_{2}$. To do this, we considered spherical planetesimals with the bulk of density $\rho$. Thus,

$$
M_{D 1, D 2}=\int_{D_{1}}^{D_{2}} \frac{4}{3} \pi \rho\left(\frac{D}{2}\right)^{3} d N(>D)=\int_{D_{1}}^{D_{2}} \frac{4}{3} \pi \rho\left(\frac{D}{2}\right)^{3} \gamma D^{-q-1} d D .
$$

We assume that our test particles with $D=1,000 \mathrm{~km}$ represent all planetesimals with size larger than $500 \mathrm{~km}$. The particles whose distribution was computed assuming $D=100 \mathrm{~km}$ represent the 
planetesimals between 30 and $500 \mathrm{~km}$, those with $D=10 \mathrm{~km}$ represent planetesimals between 3 and $30 \mathrm{~km}$ and those with $D=1 \mathrm{~km}$ represent particles with $D<3 \mathrm{~km}$. Defining $N_{o}$ the number of planetesimals with $D>500 \mathrm{~km}$, the size distribution reported above defines the number of planetesimals in each size interval. The cumulative distribution is then converted into an incremental distribution and the total mass is computed assuming a bulk density of $3 \mathrm{~g} / \mathrm{cm}^{3}$. With this procedure, the mass that we find is proportional to $N_{0}$, the number of bodies larger than $500 \mathrm{~km}$. The value of $N_{0}$ is then found imposing a total of 24 Earth masses for the disk. The resulting numbers for $N_{0}, N(>100 \mathrm{~km}), N(>10 \mathrm{~km})$ and $N(>1 \mathrm{~km})$ are: $6200,1.5872 \times 10^{7}, 1.5872 \times 10^{9}, 1.5872 \times 10^{12}$, in good agreement with Fig. 15 of Nesvorný \& Vokrouhlický (2016). The total number of particles that we have at each size at the end of the simulations reported in the previous section is of course much smaller than the real number of planetesimals in the disk. In one case for example, we have 564, 605, 629, 637 particles with a diameter of 1000, 100, 10 and $1 \mathrm{~km}$ respectively at the end of the gas phase. Thus, we created "super-particles", with a mass equal to the total mass in the considered size interval, divided by the number of particles that survived in the end of our simulations.

With the mass of the planetesimal disk in hands, the next step is to perform the simulations of the evolution of the planetary system under the effects of the different planetesimal disks showed in the section 2.3. However, for a smooth transition from the previous simulation (that featured the planets and test particles) we grow the masses of the particles from 0 to their final mass $\left(M_{f}\right)$ with a function

$$
M(t)=M_{f}(1-\exp (-t / 3 M y))
$$

In this way, planetesimals are growing their mass slowly and smoothly until reach the final SuperParticles mass after $3 \mathrm{Myr}$ of simulation. This procedure was followed to avoid an abrupt transition from a massless disk to a massive disk, which could cause artificial instabilities.

In Figure 24, we show two snapshots of one of our simulations (the one starting from the endstate of the simulation with Jupiter migrating from 10 to $5 \mathrm{AU}$ ) where the planets interact with the planetesimal disk. The color box represents the super-particles' mass in Pluto's masses. At time 3 My (Fig. 24 b) the particles have their final mass. Note that the most massive super-particles (red) correspond to the test particles with $D=100 \mathrm{~km}$ in the simulations of the previous section because most of the mass in the Nesvorný \& Vokrouhlický (2016) distribution falls in the $30<D<500 \mathrm{~km}$ range, that is represented by our $\mathrm{D}=100 \mathrm{~km}$ Super-Particles. The group of particles most dynamically excited (orange) correspond to super-particles with masses of 3 Pluto's mass and D=1000 km. The green super-particles correspond instead to Super particles with $\mathrm{D}=1 \mathrm{~km}$, strongly damped by gas drag during the planet formation-migration phase. The purple super-particles represent the superparticles with $\mathrm{D}=10 \mathrm{~km}$ with mass around of 2.4 Pluto's mass.

Because our super-particles are quite massive (several Pluto masses), when they encounter the planets they can force the latter to have spuriously large orbital jumps, that favor the rapid onset of instability. For this reason, following Gomes et al. (2005) when super-Particles come close enough to Neptune's orbit (i.e. their perihelion distance is within 2.85 Neptune's Hill radii from Neptune's aphelion distance), they are cloned 18, 30 or 150 times depending on the size of the super-Particles. The clones have all initially the same position and slightly different velocities. In this way, Neptune encounters only particles individually with 10 percent of the mass of Pluto, avoiding artificially large orbital jumps. 

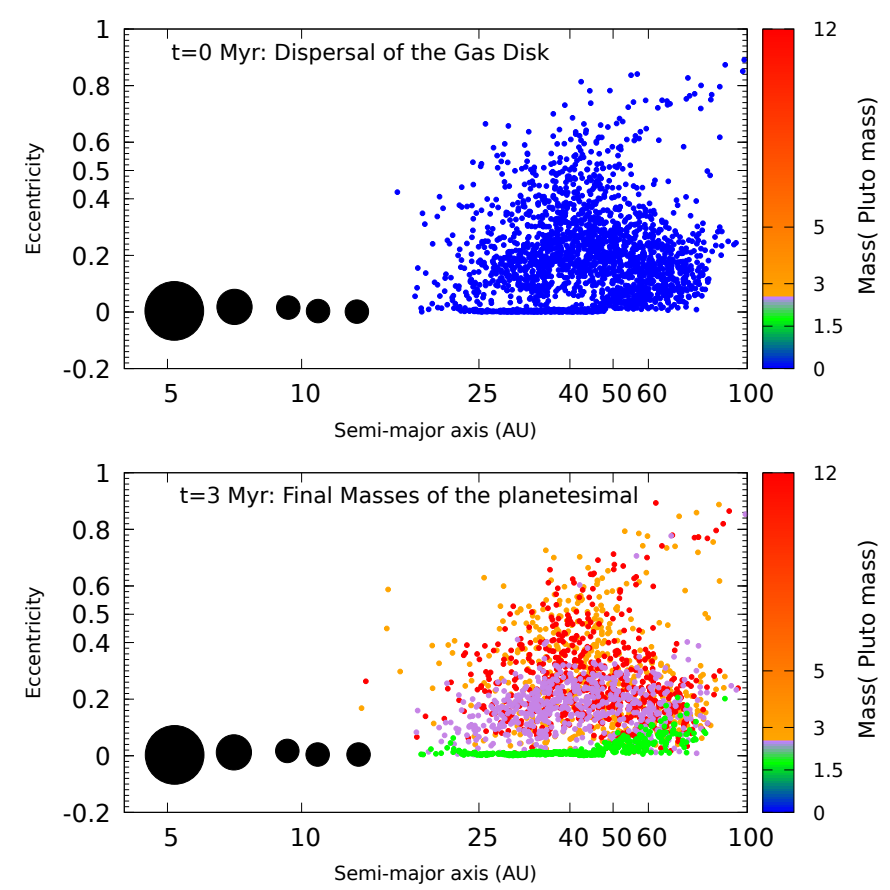

Figure 24. Two snapshots of our simulations with giant planets interacting with the planetesimal disk, starting from the end-state of the simulation with Jupiter migrating from 10 to 5 AU (Fig. 13) The color scale represents the super-particles' mass in units of Pluto's mass.

Neptune is always defined as the outermost ice giant in our planetary systems. Let $q_{N}$ be the pericenter of Neptune and $q_{p}$ the pericenter of a planetesimal. Let $\delta=\frac{5}{30} q_{\text {Neptune }}$, we clone the planetesimals when $q_{N}-q_{p}<=\delta$. In this condition, a new planetesimal is created with small deviations in the velocity of the particle to be cloned. The positions of the new planetesimals were kept fixed. In Fig. 25, we show the production of clones in four snapshots of one of our simulations with the planetesimal disc. These snapshots represent the pericenter of the planets and planetesimals as a function of the semimajor axis. At the start, the planetesimal that are already close to Neptune's orbit are immediately cloned. The new planetesimals are clustered around Neptune's pericente liner (gray line).

In this way, Neptune only undergoes close encounters with particles with masses of 10 percent the mass of Pluto, avoiding artificial jumps. The goal of this procedure was to avoid numerically inducing early instabilities. If instabilities take place in our simulated systems with 0.1 Pluto-mass planetesimals, then they should certainly have taken place in real systems with Pluto-mass planetesimals (e.g., Quarles and Kaib 2019).

\subsubsection{Statistical analyses for Planetesimal disk-triggered Instability}

Once again, we statistically studied the stability of the same 27 giant planet system from Izidoro et al. (2015a) which we used in Section 3.1 but now making use of the planetesimal disks produced 


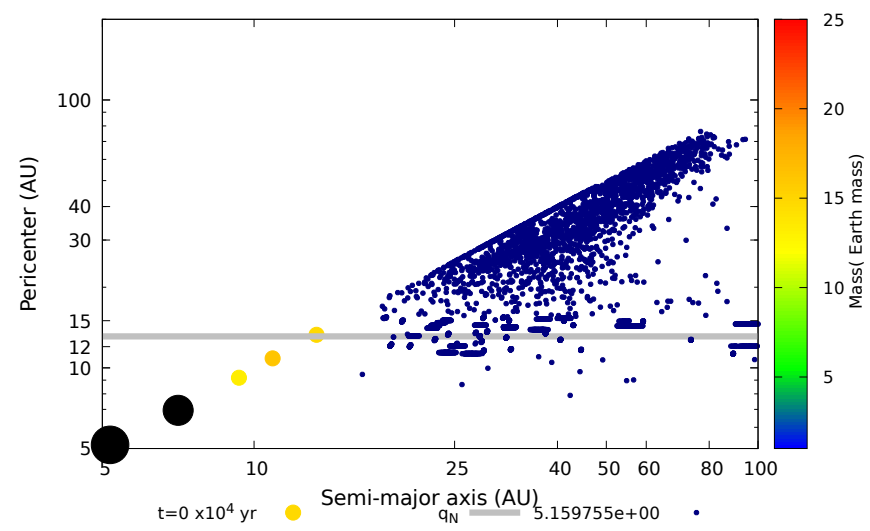

(a)

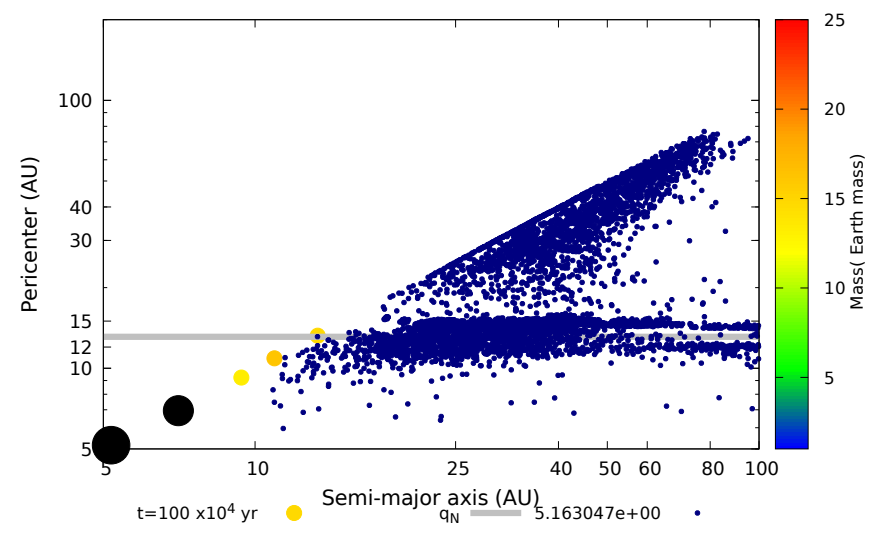

(b)
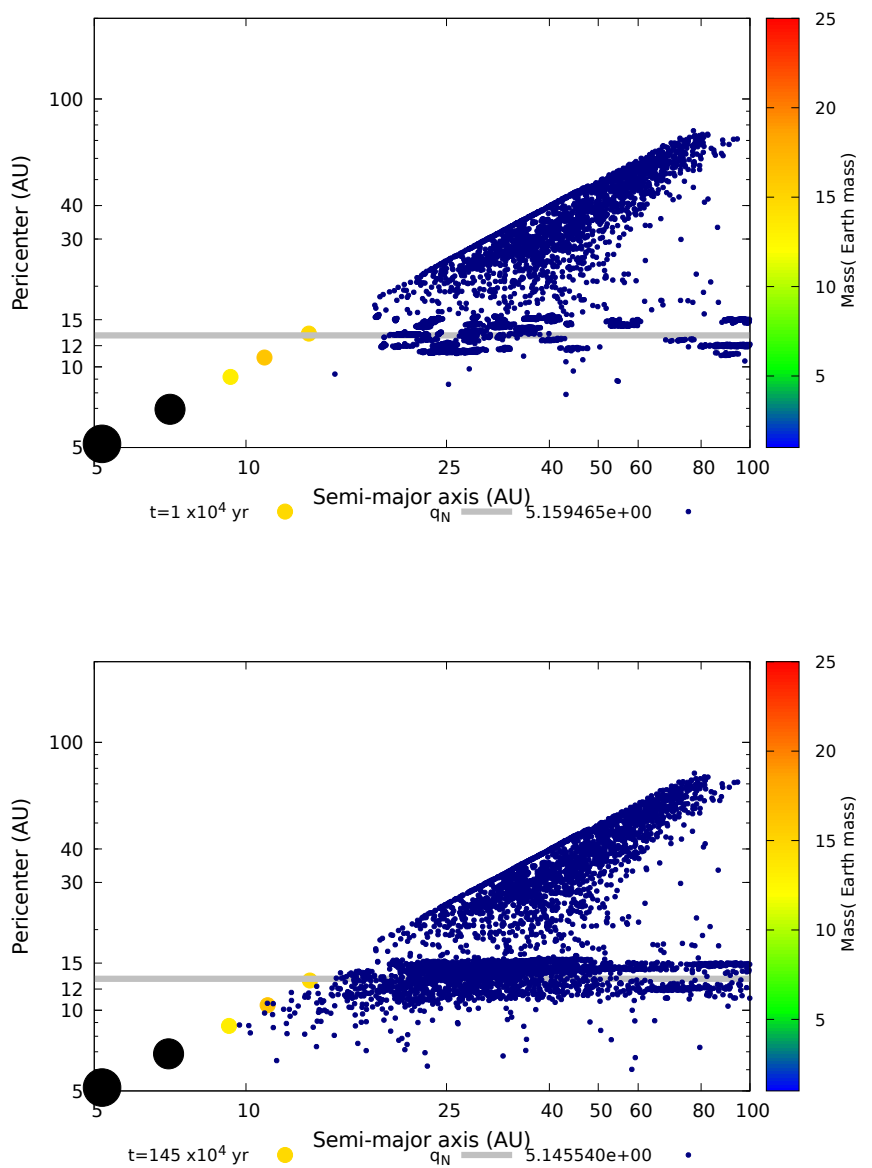

(b)

Figure 25. Evolution of the pericenter of the planets and planetesimals as a function of the semimajor axis. Clone production takes place around Neptune's pericenter line (gray line). Panel (a) represents the initial moment of the simulation and the pericenter of the planets and planetesimals in 10,000, 1 million years and 1.45 million years are showed in panels $(\mathrm{b}),(\mathrm{c})$ and $(\mathrm{d})$ respectively.

by the cases Jup_static, Jup_outward, Jup_10AU_in and Jup_15AU_in ${ }^{2}$. It is important to say we used the same planetesimals' disks from the different migrition histories for Jupiter for all 27 planetary systems. However, all of the planetesimals' disks are produced from a single case of formation of Uranus and Neptune. To maintain the same semimajor axis relative to the outermost ice giant we scale the planetesimals' orbits for each planetary system. To generate a distribution of outcomes, we performed 10 simulations of each of the 27 cases randomizing the orbital angles of the planetesimals' orbits. We have done a total of 1080 simulations. For the planets, the initial conditions were the orbital elements of the planets at the end-time of the gas phase from Izidoro et al. (2015a).

The outcome of these simulations is shown in Fig. 26. The distribution of instability times is shown separately: first we used only the self-stable systems which are stable for 1 Gy in case without any

${ }^{2}$ We did not perform simulations with the case Jup_20AU_in because of computational resources and also it is inconsistent with the primordial Kuiper Belt. (See in Section 2.4) 


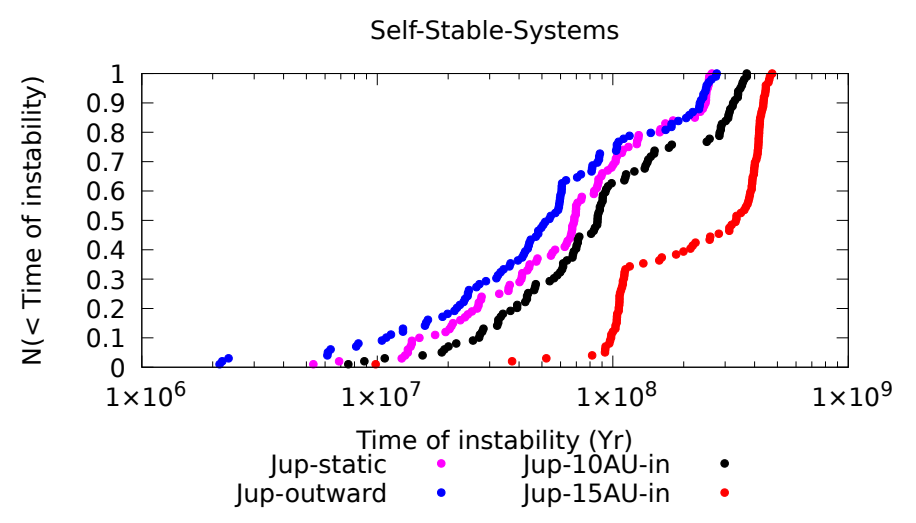

(a)

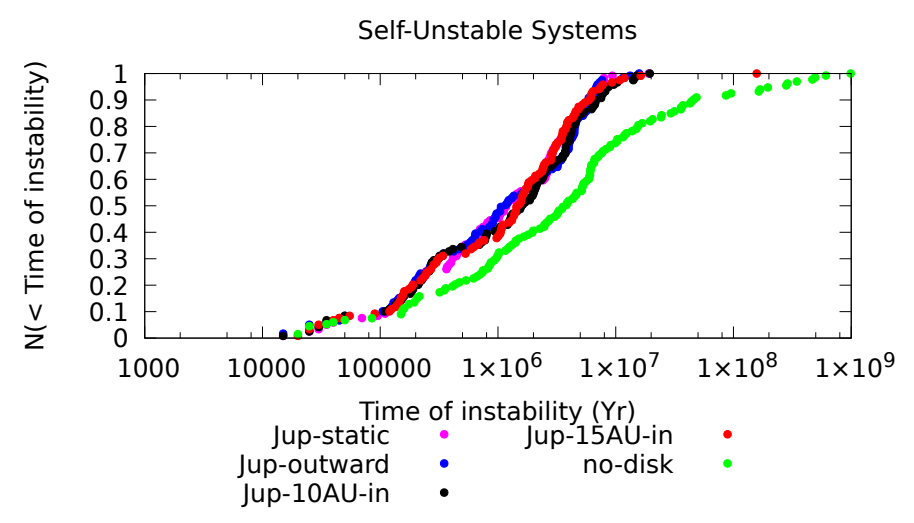

(b)

Figure 26. Cumulative normalized distributions of the timing of instabilities for the cases calculated during our simulations after the gas dispersal using the planetesimal disks produced by the cases Jup_static, Jup_outward, Jup_10AU_in and Jup_15AU_in, in pink, blue, black and red respectively. In the Panel (a) we only considered self-stable systems (i.e systems that are stable for 1 Gy without any planetesimal disk). In the Panel (b) we used the unself-stable systems (i.e systems that are unstable in less than 1 Gy without any planetesimal disk) to calculate the distribution of time of instabilities. The green points represent the distribution of unself-stable systems getting unstable without any planetesimal disk for a direct comparison.

planetesimal disk (Fig. 26 (a)) and calculated the distribution times using the planetesimal disks; and second we used only the unself-stable system which are those systems unstable in less than 1 Gy in case of any planetesimal disk (Fig. 26 (b)) to calculate the distribution times now using the planetesimal disks. For comparison with the results of Section 3.1 while $48 \%$ of simulations without planetesimal disks were stable for 1 billion years without planetesimal disks, all of the simulations with disks were unstable within 500 Myr.

According with the Panel (a) of Fig. 26, as we expected, the fraction of stable system depends of the initial distance between Neptune and the inner border of the disk. For example, the case with Jupiter on a non-migrating orbit at 5 AU results in a median instability time of $60 \mathrm{My}$, whereas the case where Jupiter migrates from 15 to $5 \mathrm{AU}$, which results in a much wider separation between Neptune and the inner edge of the disk, results in a median instability time of $134.70 \mathrm{My}$. These results were predicted by Deienno et al. (2017) based on the planetesimal disc results from sect. 2.2 and are broadly consistent with the results of Quarles and Kaib (2019). Although we have seen in Fig. 22 that the case with Jupiter migrating from 10 to 5 AU overexcites the cold Kuiper belt, Fig. 26 (a) shows that the distribution of instability times in that case is similar to that of no-Jupitermigration case. So we retain this distribution as an upper bound of the instability times of acceptable short-range inward migration cases. Thus we conclude that the median instability times range in the interval 50.0-80.3My, with $75 \%$ of the instabilities occurring within $190 \mathrm{My}$.

For the unself-stable systems (Panel (b) of Fig. 26) the times of instabilities, in general, are not dependent on the way that Jupiter migrated. Thus, the distribution of the time of instabilities is not dependent on the gap between the disk and the planets. However, the median of instability for the four cases with the planetesimal disk is $1.5 \mathrm{My}$ with $99 \%$ of the all unself-stable systems go unstable in less than 10 My. Only $75 \%$ of the unself-stable systems go unstable in less than 10 My without 
any planetesimal disk (green points in Panel (b) Fig. Fig. 26). It shows that the planetesimal disk plays an important role to drive the instabilities to early times.

\section{DISCUSSION}

Our results strongly favor an early giant planet instability. A natural outcome of successful ice giant formation simulations (Izidoro et al. 2015a) is a self-triggered instability within $10 \mathrm{Myr}$ of the dissipation of the disk (§3.1). If instead the giant planets emerged from the disk on stable orbits, then interactions with the planetesimal disk should nonetheless have triggered the instability within 500 Myr. If we discard the cases where Jupiter migrated inwards by $5 \mathrm{AU}$ or more because they result in a cold Kuiper belt that is too dynamical excited (Fig. 26), the instability times are within 200My, of which $70 \%$ are within $70 \mathrm{My}$. In this section we discuss how our result fits within a broader context. First we compare our dynamically-inferred timeframe with empirical constraints for the giant planet instability (§4.1). Finally, we present the limitations of our simulations (§4.2) to motivate future work that might improve the results.

\subsection{Empirical Constraints}

The division between an "early" and a "late" instability remains fuzzy. This is essential in understanding the timeline of Solar System evolution in the context of other landmarks. For instance, the Sun's gaseous disk dissipated 3-5 Myr after CAIs (calcium-aluminium-rich inclusion, probably first solids to condensate in the sun's natal disk), judging from the timescale for the disappearance of disks around other young stars (Haisch et al. 2001; Pascucci et al. 2009) as well as the ages of the oldest chondrules (e.g. Krot et al. 2005; Bollard et al. 2015). Mars' growth was complete on a similar timescale of 5-10 Myr (Nimmo \& Kleine 2007; Dauphas \& Pourmand 2011), whereas the Moon-forming impact did not occur until roughly 50-100 Myr later (e.g., review by Kleine et al. 2009).

Morbidelli et al. (2018) showed that the lunar cratering record and the highly-siderophile element abundances of the Earth and Moon could be reconciled by an instability that happened in the first hundred million years of Solar System history, provided that the highly siderophile elements have been removed from the lunar mantle during a late crystallization of the lunar magma ocean. Other re-analyses of the cratering record and the age distribution of Apollo samples also point to an early instability, but without firm timing constraints (Boehnke \& Harrison 2016; Zellner 2017; Michael et al. 2018).

Some dynamical analyses have derived more quantitative estimates. An instability within $100 \mathrm{Myr}$ after CAIs is required to explain the survival of the Patroclus-Menoetius Jupiter binary Trojan, assuming it was formed in the primordial Kuiper belt and dynamically captured during the instability (Nesvorný et al. 2018). Nesvorný (2015a) found that the instability could not have happened earlier than $\sim 10$ Myr by arguing that Neptune's slow, pre-instability migration is needed to excite the inclinations of Kuiper belt objects. Morbidelli and Nesvorny (submitted, 2019) found that the current size-distribution of the Kuiper belt could be obtained starting from the size distribution coming from streaming-instability models (Simon et al. 2016) provided that the trans-Neptunian planetesimal disk was not dispersed before $\sim 50 \mathrm{My}$.

On the other hand, a very early instability occurring before the completion of the accretion of the terrestrial planets would bypasse the problem of the dynamical fragility of the terrestrial planet system (Brasser et al. 2009; Agnor \& Lin 2012; Kaib \& Chambers 2016). Moreover, such an early 
instability could have removed most of the material from Mars's formation region, explaining the small mass of Mars and its short accretion timescale (Clement et al. 2018a). It could have also contributed to depleting and dynamically exciting the asteroids (Morbidelli et al. 2010; Nesvorný et al. 2017; Deienno et al. 2018; Clement et al. 2019a). These considerations seem to favor an instability within the first $\sim 10 \mathrm{My}$, possibly even less.

However an instability before the Moon-forming impact - generally thought to be the last giant impact in the inner Solar System - should have left a chemical imprint on Earth's interior that is not observed. In fact, the isotopic signatures of atmospheric and mantle Xenon are distinct (Caracausi et al. 2016). Marty et al. (2017) used the isotopic signature of comet 67P measured by the ROSETTA spacecraft to argue that $\sim 20 \%$ of present-day atmospheric Xenon is of cometary origin. This naively suggests that the cometary bombardment (necessarily associated to the giant planet instability) occurred after the formation of the Earth's crust, which would imply a giant planet instability later than the Moon-forming impact, no earlier than 50-100 Myr after CAIs (e.g., Kleine et al. 2009). However, if the in-gassing of Xenon in the silicate magma was inefficient, cometary Xenon may not have penetrated into the terrestrial mantle during the magma-ocean phase that followed the giant impact. It is also known that a large fraction of the atmosphere of the proto-Earth might have survived the giant impact, particulalry if there was no surface ocean at that time (Schlichting \& Mukhopadhyay 2018). Thus, it may be possible that the cometary bombardment predated the Moon-forming event, although this requires further geochemical investigations.

In conclusions, it is not possible to conclude from firm constraints when the giant planet instability occurred within the first $100 \mathrm{My}$. Unfortunately, the present study, with a median instability time of 36.78-61.5 My and a 75\% instability time of $136 \mathrm{My}$ when excluding the cases with long-range inward migration of Jupiter does not help in assessing what is the most probable time.

\subsection{Limitations of our work}

As with any numerical study, our simulations are a simplified and idealized version of reality. One main limitation is that our results are based on a small number of outcomes from Izidoro et al. (2015a). Nevertheless, these simulations represent, to our knowledge, the only models that quantitatively explain the origin of Uranus and Neptune. We performed N-body simulation of Jupiter, Saturn and a small number of planetary embryos from Izidoro et al. (2015a) simulations. Thus, we artificially forced Jupiter to migrate and rescaled the semi-major axes of the others planets and embryos from the original integration to Jupiter's. The evolution of the other elements, for example, eccentricities and inclinations is kept the same as in the original simulation. This procedure is not ideal. Even if all bodies were locked in mutual mean motion resonances, the global migration of the system would affect the orbital eccentricities. However, in all cases the eccentricities should be small (of order $h^{2}$, where $\mathrm{h}$ is the aspect ratio of the disk). We expect that the slightly different eccentricities considering different migration paths for Jupiter would not have dramatic consequences on the resulting dynamical structure of the planetesimal disk. Instead, the dynamical sculpting of the planetesimals disk depends mainly on how long the embryos stay in the disk and therefore on the migration of the whole chain (determined by the migration of Jupiter). A more realistic model would require very computationally costly hydrodynamical simulations. The advantage of our procedure is that we can compare the results obtained imposing just different migration patterns for Jupiter, keeping all other parameters equal. If we had done different simulations for each migration pattern 
of Jupiter, too many aspects of the evolution would have changed (due to the fact that all evolutions are chaotic), making it difficult to determine what was the cause of the different final results.

Our study of the stability of self-triggered and planetesimal disk-triggered simulations included a total of 1080 simulations whereas our self-consistent planetesimal disks were generated from a single simulation of Uranus and Neptune formation. Pebble accretion can explain the rapid growth of super-Earths and Ice giants in different parts of the gas-disk including the region inside of the cold Kuiper Belt (or the current trans-Neptunian region (Lambrechts \& Johansen 2014; Johansen \& Lambrechts 2017; Izidoro et al. 2019; Bitsch et al. 2019; Lambrechts et al. 2019)). Other formation histories are possible. For example, Jupiter could have formed far out, then Saturn and embryos form in sequence, in the same location once the previous planet has migrated away. In that case, the cold Kuiper belt would be much less excited, even in the case of an initial distant location of Jupiter (e.g. 20 AU). Our result show actually how far out the formation of embryos could have ocurred before exciting the cold Kuiper belt too much, rather than how far out Jupiter could form. Given that our simulations are built upon those of Izidoro et al. (2015a), we inherit the limitations of that study. For example, as discussed above, our model for the underlying gaseous disk is plausible but uncertain. In addition, we did not include potentially important processes such as collisional fragmentation (Leinhardt \& Stewart 2012; Genda et al. 2012) and pebble accretion (Johansen \& Lambrechts 2017). Our simulations also start with an already-formed Jupiter and Saturn. If their growth dramatically re-shaped the outer Solar System then our initial conditions would have to be re-thought.

\section{CONCLUSIONS}

In this paper we broadly constrained the timing of the giant planet instability using simulations of the early evolution of the outer Solar System. We started from the best simulations of Izidoro et al. (2015a) of the growth of the ice giants during the gaseous disk phase via inward migration of $\sim 5 M_{\oplus}$ cores that are blocked by the already-formed Jupiter and Saturn. We generated plausible outer planetesimal disks that were dynamically sculpted in a self-consistent way during this formation process, taking into account both the size-dependence of aerodynamic gas drag and a range of possible migration histories for Jupiter and Saturn (see Pierens et al. 2014b). We determined that a large fraction $(\sim 50 \%)$ of the giant planet configurations generated by Izidoro et al. (2015a) became unstable within $10 \mathrm{Myr}$ of disk dissipation. The leads to the possibility that the giant planet instability was self-triggered by the planets themselves, which is new in the context of the Solar System's history but a well-known process in a more general context (e.g. Chambers et al. 1996; Marzari \& Weidenschilling 2002; Ford \& Rasio 2008).

When we introduce the different outer planetesimal disks, the giant planet configurations that would have remained stable if they had been alone went unstable within $500 \mathrm{Myr}$, and generally much faster. The median instability timescale is 36.78-61.5 My. If we exclude the disks sculpted during long-range inward migrations of Jupiter, which are inconsistent with the small dynamical excitaiton of the cold Kuiper belt, the instability time is within $136 \mathrm{My}$ in $75 \%$ of the cases, which is consistent with the conclusions of Nesvorný et al. (2018) on the survival of the Trojan Patroclos as primordial binary from the Kuiper belt. Unfortunately, given our statistics and the available constraints, it is difficult to conclude when the giant planet instability happened within the first $\sim 100 \mathrm{My}$. In particular it is difficult to assess whether the giant planet instability pre-dates or post-dates the Moon-forming event. 


\section{ACKNOWLEDGMENTS}

We acknowledge helpful discussions with Sarah Stewart on the origin of Earth's Xenon. We also thank John E. Chambers and Rogerio Deienno for useful comments and suggestions on the submitted manuscript. R.R.S acknowledges support provided by grants \#2017/09919-8, \#2016/24561-0 and \#2015/15588-9, São Paulo Research Foundation (FAPESP) and the good hospitality received from Observatoire de la Côte d'Azur. A.I. thanks finnancial support from Fapesp via grants \#2016/126862 and \#2016/19556-7. S. N. R. thanks the Agence Nationale pour la Recherche for funding via grant ANR-13-BS05-0003-002 (grant MOJO).

\section{REFERENCES}

Adams, F. C., \& Laughlin, G. 2003, Icarus, 163, 290, doi: 10.1016/S0019-1035(03)00081-2

Agnor, C. B., \& Lin, D. N. C. 2012, ApJ, 745, 143, doi: 10.1088/0004-637X/745/2/143

Batalha, N. M., Rowe, J. F., Bryson, S. T., et al. 2013, ApJS, 204, 24, doi: $10.1088 / 0067-0049 / 204 / 2 / 24$

Bitsch, B., Lambrechts, M., \& Johansen, A. 2015, A\&A, 582, A112, doi: 10.1051/0004-6361/201526463

Bitsch, B., Raymond, S. N., \& Izidoro, A. 2019, A\&A, 624, A109, doi: 10.1051/0004-6361/201935007

Boehnke, P., \& Harrison, T. M. 2016, Proceedings of the National Academy of Science, 113, 10802, doi: 10.1073/pnas.1611535113

Bollard, J., Connelly, J. N., \& Bizzarro, M. 2015, Meteoritics and Planetary Science, 50, 1197, doi: 10.1111/maps.12461

Bottke, W. F., Vokrouhlický, D., Minton, D., et al. 2012, Nature, 485, 78, doi: 10.1038/nature10967

Boué, G., \& Laskar, J. 2010, The Astrophysical Journal Letters, 712, L44. http: //stacks.iop.org/2041-8205/712/i=1/a=L44

Boué, G., Laskar, J., \& Kuchynka, P. 2009, ApJL, 702, L19, doi: 10.1088/0004-637X/702/1/L19

Brasser, R., Duncan, M. J., \& Levison, H. F. 2007, Icarus, 191, 413, doi: 10.1016/j.icarus.2007.05.003

Brasser, R., \& Lee, M. H. 2015, AJ, 150, 157, doi: 10.1088/0004-6256/150/5/157

Brasser, R., Matsumura, S., Ida, S., Mojzsis, S. J., \& Werner, S. C. 2016, ApJ, 821, 75, doi: 10.3847/0004-637X/821/2/75

Brasser, R., Morbidelli, A., Gomes, R., Tsiganis, K., \& Levison, H. F. 2009, A\&A, 507, 1053, doi: 10.1051/0004-6361/200912878
Capobianco, C. C., Duncan, M., \& Levison, H. F. 2011, Icarus, 211, 819, doi: 10.1016/j.icarus.2010.09.001

Caracausi, A., Avice, G., Bernard, P., Furi, E., \& Marty, B. 2016, in EGU General Assembly Conference Abstracts, Vol. 18, EGU General Assembly Conference Abstracts, EPSC2016-16723

Chambers, J. E., Wetherill, G. W., \& Boss, A. P. 1996, Icarus, 119, 261, doi: 10.1006/icar.1996.0019

Chatterjee, S., Ford, E. B., Matsumura, S., \& Rasio, F. A. 2008, ApJ, 686, 580, doi: $10.1086 / 590227$

Clement, M. S., Kaib, N. A., Raymond, S. N., Chambers, J. E., \& Walsh, K. J. 2018a, ArXiv e-prints

—. 2019a, Icarus, 321, 778, doi: 10.1016/j.icarus.2018.12.033

Clement, M. S., Kaib, N. A., Raymond, S. N., \& Walsh, K. J. 2018b, Icarus, 311, 340, doi: 10.1016/j.icarus.2018.04.008

Clement, M. S., Raymond, S. N., \& Kaib, N. A. 2019b, AJ, 157, 38, doi: 10.3847/1538-3881/aaf21e

Cresswell, P., \& Nelson, R. P. 2006, A\&A, 450, 833, doi: 10.1051/0004-6361:20054551

—. 2008, A\&A, 482, 677, doi: 10.1051/0004-6361:20079178

Crida, A., \& Bitsch, B. 2017, Icarus, 285, 145, doi: 10.1016/j.icarus.2016.10.017

Crida, A., \& Morbidelli, A. 2007, MNRAS, 377, 1324, doi: 10.1111/j.1365-2966.2007.11704.x

Dauphas, N., \& Pourmand, A. 2011, Nature, 473, 489, doi: 10.1038/nature10077 
Davies, M. B., Adams, F. C., Armitage, P., et al. 2014, Protostars and Planets VI, 787, doi: 10.2458/azu_uapress_9780816531240-ch034

Day, J. M. D., Pearson, D. G., \& Taylor, L. A. 2007, Science, 315, 217, doi: $10.1126 /$ science. 1133355

Day, J. M. D., \& Walker, R. J. 2015, Earth and Planetary Science Letters, 423, 114, doi: 10.1016/j.epsl.2015.05.001

de Sousa, R. R., Gomes, R., Morbidelli, A., \& Neto, E. V. 2018, Icarus, doi: https: //doi.org/10.1016/j.icarus.2018.08.008

Deienno, R., Gomes, R. S., Walsh, K. J., Morbidelli, A., \& Nesvorný, D. 2016, Icarus, 272, 114, doi: 10.1016/j.icarus.2016.02.043

Deienno, R., Izidoro, A., Morbidelli, A., et al. 2018, ApJ, 864, 50, doi: $10.3847 / 1538-4357 / \operatorname{aad} 55 \mathrm{~d}$

Deienno, R., Morbidelli, A., Gomes, R. S., \& Nesvorný, D. 2017, AJ, 153, 153, doi: 10.3847/1538-3881/aa5eaa

Deienno, R., Yokoyama, T., Nogueira, E. C., Callegari, N., \& Santos, M. T. 2011, A\&A, 536, A57, doi: 10.1051/0004-6361/201014862

Dones, L., \& Tremaine, S. 1993, Icarus, 103, 67, doi: 10.1006/icar.1993.1059

Elkins-Tanton, L. T., Burgess, S., \& Yin, Q.-Z. 2011, Earth and Planetary Science Letters, 304, 326, doi: 10.1016/j.epsl.2011.02.004

Fogg, M. J., \& Nelson, R. P. 2005, A\&A, 441, 791, doi: 10.1051/0004-6361:20053453

Ford, E. B., \& Rasio, F. A. 2008, ApJ, 686, 621, doi: 10.1086/590926

Fraser, W. C., Brown, M. E., Morbidelli, A., Parker, A., \& Batygin, K. 2014, ApJ, 782, 100, doi: 10.1088/0004-637X/782/2/100

Genda, H., Kokubo, E., \& Ida, S. 2012, ApJ, 744, 137, doi: 10.1088/0004-637X/744/2/137

Goldreich, P., \& Tremaine, S. 1980, ApJ, 241, 425, doi: $10.1086 / 158356$

Gomes, R., Levison, H. F., Tsiganis, K., \& Morbidelli, A. 2005, Nature, 435, 466, doi: $10.1038 /$ nature 03676

Gomes, R., \& Nesvorný, D. 2016, A\&A, 592, A146, doi: 10.1051/0004-6361/201527757

Gomes, R., Nesvorný, D., Morbidelli, A., Deienno, R., \& Nogueira, E. 2018, Icarus, 306, 319, doi: $10.1016 /$ j.icarus.2017.10.018

Grishin, E., \& Perets, H. B. 2015, ApJ, 811, 54, doi: 10.1088/0004-637X/811/1/54
Haisch, Jr., K. E., Lada, E. A., \& Lada, C. J. 2001, ApJL, 553, L153, doi: 10.1086/320685

Hamilton, D. P., \& Ward, W. R. 2004, AJ, 128, 2510, doi: 10.1086/424534

Hartmann, W. K. 1975, Icarus, 24, 181, doi: 10.1016/0019-1035(75)90095-0

—. 2003, Meteoritics and Planetary Science, 38, 579, doi: 10.1111/j.1945-5100.2003.tb00028.x

Hillenbrand, L. A. 2008, Physica Scripta Volume $\mathrm{T}, 130,014024$, doi: 10.1088/0031-8949/2008/T130/014024

Izidoro, A., Bitsch, B., Raymond, S. N., et al. 2019, arXiv e-prints, arXiv:1902.08772. https://arxiv.org/abs/1902.08772

Izidoro, A., Morbidelli, A., Raymond, S. N., Hersant, F., \& Pierens, A. 2015a, A\&A, 582, A99, doi: 10.1051/0004-6361/201425525

Izidoro, A., Ogihara, M., Raymond, S. N., et al. 2017, MNRAS, 470, 1750, doi: 10.1093/mnras/stx1232

Izidoro, A., Raymond, S. N., Morbidelli, A., Hersant, F., \& Pierens, A. 2015b, ApJL, 800, L22, doi: 10.1088/2041-8205/800/2/L22

Jakubík, M., Morbidelli, A., Neslušan, L., \& Brasser, R. 2012, A\&A, 540, A71, doi: 10.1051/0004-6361/201117687

Johansen, A., \& Lacerda, P. 2010, MNRAS, 404, 475, doi: 10.1111/j.1365-2966.2010.16309.x

Johansen, A., \& Lambrechts, M. 2017, Annual Review of Earth and Planetary Sciences, 45, 359, doi: 10.1146/annurev-earth-063016-020226

Kaib, N. A., \& Chambers, J. E. 2016, MNRAS, 455, 3561, doi: 10.1093/mnras/stv2554

Kegerreis, J. A., Teodoro, L. F. A., Eke, V. R., et al. 2018, ApJ, 861, 52, doi: $10.3847 / 1538-4357 /$ aac725

Kleine, T., Touboul, M., Bourdon, B., et al. 2009, GeoCoA, 73, 5150

Krot, A. N., Amelin, Y., Cassen, P., \& Meibom, A. 2005, Nature, 436, 989, doi: $10.1038 /$ nature03830

Lambrechts, M., \& Johansen, A. 2012, A\&A, 544, A32, doi: 10.1051/0004-6361/201219127

—. 2014, A\&A, 572, A107, doi: 10.1051/0004-6361/201424343

Lambrechts, M., Johansen, A., \& Morbidelli, A. 2014, A\&A, 572, A35, doi: 10.1051/0004-6361/201423814 
Lambrechts, M., Morbidelli, A., Jacobson, S. A., et al. 2019, A\&A, 627, A83, doi: 10.1051/0004-6361/201834229

Leinhardt, Z. M., \& Stewart, S. T. 2012, ApJ, 745, 79, doi: 10.1088/0004-637X/745/1/79

Levison, H. F., Kretke, K. A., \& Duncan, M. J. 2015, Nature, 524, 322, doi: $10.1038 /$ nature 14675

Levison, H. F., Morbidelli, A., Tsiganis, K., Nesvorný, D., \& Gomes, R. 2011, AJ, 142, 152, doi: 10.1088/0004-6256/142/5/152

Levison, H. F., \& Stewart, G. R. 2001, Icarus, 153, 224, doi: 10.1006/icar.2001.6672

Levison, H. F., Thommes, E., \& Duncan, M. J. 2010, AJ, 139, 1297, doi: 10.1088/0004-6256/139/4/1297

Lin, D. N. C., \& Ida, S. 1997, ApJ, 477, 781, doi: $10.1086 / 303738$

Marcy, G. W., Isaacson, H., Howard, A. W., et al. 2014, ApJS, 210, 20, doi: 10.1088/0067-0049/210/2/20

Marty, B., Altwegg, K., Balsiger, H., et al. 2017, Science, 356, 1069, doi: 10.1126/science.aal3496

Marzari, F., \& Weidenschilling, S. J. 2002, Icarus, 156, 570, doi: 10.1006/icar.2001.6786

Masset, F., \& Snellgrove, M. 2001, MNRAS, 320, L55, doi: 10.1046/j.1365-8711.2001.04159.x

Matsumoto, Y., Nagasawa, M., \& Ida, S. 2012, Icarus, 221, 624, doi: $10.1016 /$ j.icarus.2012.08.032

Matsumura, S., Thommes, E. W., Chatterjee, S., \& Rasio, F. A. 2010, ApJ, 714, 194, doi: 10.1088/0004-637X/714/1/194

Mayor, M., Marmier, M., Lovis, C., et al. 2011, arXiv:1109.2497.

https://arxiv.org/abs/1109.2497

Michael, G., Basilevsky, A., \& Neukum, G. 2018, Icarus, 302, 80, doi: 10.1016/j.icarus.2017.10.046

Moeckel, N., Raymond, S. N., \& Armitage, P. J. 2008, ApJ, 688, 1361, doi: 10.1086/592286

Morbidelli, A., Brasser, R., Gomes, R., Levison, H. F., \& Tsiganis, K. 2010, AJ, 140, 1391, doi: 10.1088/0004-6256/140/5/1391

Morbidelli, A., \& Crida, A. 2007, Icarus, 191, 158, doi: 10.1016/j.icarus.2007.04.001

Morbidelli, A., Levison, H. F., Tsiganis, K., \& Gomes, R. 2005, Nature, 435, 462, doi: $10.1038 /$ nature 03540
Morbidelli, A., Marchi, S., Bottke, W. F., \& Kring, D. A. 2012a, Earth and Planetary Science Letters, 355, 144, doi: 10.1016/j.epsl.2012.07.037

Morbidelli, A., Nesvorny, D., Laurenz, V., et al. 2018, Icarus, 305, 262, doi: 10.1016/j.icarus.2017.12.046

Morbidelli, A., Tsiganis, K., Batygin, K., Crida, A., \& Gomes, R. 2012b, Icarus, 219, 737, doi: $10.1016 /$ j.icarus.2012.03.025

Morbidelli, A., Tsiganis, K., Crida, A., Levison, H. F., \& Gomes, R. 2007, AJ, 134, 1790, doi: $10.1086 / 521705$

Nesvorný, D. 2011, ApJL, 742, L22, doi: 10.1088/2041-8205/742/2/L22

—. 2015a, AJ, 150, 73, doi: 10.1088/0004-6256/150/3/73

—. 2015b, AJ, 150, 68, doi: 10.1088/0004-6256/150/3/68

—. 2018, ARA\&A, 56, 137, doi: 10.1146/annurev-astro-081817-052028

Nesvorný, D., \& Morbidelli, A. 2012, AJ, 144, 117, doi: 10.1088/0004-6256/144/4/117

Nesvorný, D., Roig, F., \& Bottke, W. F. 2017, AJ, 153, 103, doi: $10.3847 / 1538-3881 / 153 / 3 / 103$

Nesvorný, D., \& Vokrouhlický, D. 2016, ApJ, 825, 94, doi: 10.3847/0004-637X/825/2/94

Nesvorný, D., Vokrouhlický, D., Bottke, W. F., \& Levison, H. F. 2018, Nature Astronomy, doi: 10.1038/s41550-018-0564-3

Nesvorný, D., Vokrouhlický, D., \& Deienno, R. 2014, ApJ, 784, 22, doi: 10.1088/0004-637X/784/1/22

Nesvorný, D., Vokrouhlický, D., \& Morbidelli, A. 2007, AJ, 133, 1962, doi: 10.1086/512850

—. 2013, ApJ, 768, 45, doi: 10.1088/0004-637X/768/1/45

Neukum, G., Ivanov, B. A., \& Hartmann, W. K. 2001, SSRv, 96, 55

Nimmo, F., \& Kleine, T. 2007, Icarus, 191, 497, doi: 10.1016/j.icarus.2007.05.002

Paardekooper, S. J., \& Papaloizou, J. C. B. 2008, A\&A, 485, 877, doi: 10.1051/0004-6361:20078702

Papaloizou, J. C. B., \& Larwood, J. D. 2000, MNRAS, 315, 823, doi: 10.1046/j.1365-8711.2000.03466.x

Pascucci, I., Apai, D., Luhman, K., et al. 2009, ApJ, 696, 143, doi: 10.1088/0004-637X/696/1/143 
Pierens, A., \& Nelson, R. P. 2008, A\&A, 482, 333, doi: 10.1051/0004-6361:20079062

Pierens, A., \& Raymond, S. N. 2011, A\&A, 533, A131, doi: 10.1051/0004-6361/201117451

Pierens, A., Raymond, S. N., Nesvorny, D., \& Morbidelli, A. 2014a, ApJL, 795, L11, doi: 10.1088/2041-8205/795/1/L11

—. 2014b, ApJL, 795, L11, doi: 10.1088/2041-8205/795/1/L11

Quarles, B., \& Kaib, N. 2019, AJ, 157, 67, doi: 10.3847/1538-3881/aafa71

Rasio, F. A., \& Ford, E. B. 1996, Science, 274, 954, doi: 10.1126/science.274.5289.954

Raymond, S. N., Armitage, P. J., \& Gorelick, N. 2010, ApJ, 711, 772, doi: 10.1088/0004-637X/711/2/772

Raymond, S. N., \& Izidoro, A. 2017, Icarus, 297, 134, doi: 10.1016/j.icarus.2017.06.030

Raymond, S. N., Mandell, A. M., \& Sigurdsson, S. 2006, Science, 313, 1413, doi: 10.1126/science.1130461

Raymond, S. N., \& Morbidelli, A. 2014, in IAU Symposium, Vol. 310, Complex Planetary Systems, Proceedings of the International Astronomical Union, 194-203, doi: 10.1017/S1743921314008254

Raymond, S. N., Armitage, P. J., Moro-Martín, A., et al. 2011, A\&A, 530, A62, doi: 10.1051/0004-6361/201116456

Rein, H., \& Liu, S.-F. 2012, A\&A, 537, A128, doi: 10.1051/0004-6361/201118085

Rein, H., \& Spiegel, D. S. 2015, MNRAS, 446, 1424, doi: 10.1093/mnras/stu2164

Rein, H., \& Tamayo, D. 2015, MNRAS, 452, 376, doi: $10.1093 /$ mnras/stv1257

Robert, C. M. T., Crida, A., Lega, E., Méheut, H., \& Morbidelli, A. 2018, A\&A, 617, A98, doi: 10.1051/0004-6361/201833539

Roig, F., \& Nesvorný, D. 2015, AJ, 150, 186, doi: 10.1088/0004-6256/150/6/186

Roig, F., Nesvorný, D., \& DeSouza, S. R. 2016, ApJL, 820, L30, doi: $10.3847 / 2041-8205 / 820 / 2 / L 30$

Ryder, G. 1990, EOS Transactions, 71, 313, doi: 10.1029/90EO00086
—. 2002, Journal of Geophysical Research (Planets), 107, 5022, doi: 10.1029/2001JE001583

Safronov, V. S. 1969, Evoliutsiia doplanetnogo oblaka., ed. Safronov, V. S.

Schlichting, H. E., \& Mukhopadhyay, S. 2018, Space Science Reviews, 214, 34, doi: 10.1007/s11214-018-0471-z

Shakura, N. I., \& Sunyaev, R. A. 1973, A\&A, 500, 33

Simon, J. B., Armitage, P. J., Li, R., \& Youdin, A. N. 2016, ApJ, 822, 55, doi: $10.3847 / 0004-637 \mathrm{X} / 822 / 1 / 55$

Slattery, W. L., Benz, W., \& Cameron, A. G. W. 1992, Icarus, 99, 167, doi: 10.1016/0019-1035(92)90180-F

Tanaka, H., Takeuchi, T., \& Ward, W. R. 2002, ApJ, 565, 1257, doi: 10.1086/324713

Tera, F., Papanastassiou, D. A., \& Wasserburg, G. J. 1974, Earth and Planetary Science Letters, 22, 1, doi: 10.1016/0012-821X(74)90059-4

Thommes, E. W., Duncan, M. J., \& Levison, H. F. 2002, AJ, 123, 2862, doi: 10.1086/339975

Thommes, E. W., \& Lissauer, J. J. 2003, ApJ, 597, 566, doi: 10.1086/378317

Tsiganis, K., Gomes, R., Morbidelli, A., \& Levison, H. F. 2005, Nature, 435, 459, doi: 10.1038/nature03539

Vokrouhlický, D., \& Nesvorný, D. 2015, ApJ, 806, 143, doi: 10.1088/0004-637X/806/1/143

Walker, R. J. 2009, Chemie der Erde / Geochemistry, 69, 101, doi: 10.1016/j.chemer.2008.10.001

Walsh, K. J., Morbidelli, A., Raymond, S. N., O'Brien, D. P., \& Mandell, A. M. 2011, Nature, 475, 206, doi: 10.1038/nature10201

Ward, W. R. 1986, Icarus, 67, 164, doi: 10.1016/0019-1035(86)90182-X

Ward, W. R., \& Hamilton, D. P. 2004, AJ, 128, 2501, doi: 10.1086/424533

Weidenschilling, S. J., \& Marzari, F. 1996, Nature, 384, 619, doi: 10.1038/384619a0

Wolfgang, A., Rogers, L. A., \& Ford, E. B. 2016, ApJ, 825, 19, doi: 10.3847/0004-637X/825/1/19

Zellner, N. E. B. 2017, Origins of Life and Evolution of the Biosphere, 47, 261, doi: 10.1007/s11084-017-9536-3 